ARTICLE

\title{
Determining the charge distribution and the direction of bond cleavage with femtosecond anisotropic $x$-ray liquidography
}

Jun Heo (10 1,2,3, Jong Goo Kim (1) 1,2,3, Eun Hyuk Choi ${ }^{1,2}$, Hosung Ki ${ }^{1,2}$, Doo-Sik Ahn ${ }^{1,2}$, Jungmin Kim (i) ${ }^{1,2}$, Seonggon Lee (i) ${ }^{1,2} \&$ Hyotcherl Ihee (1) ${ }^{1,2 \otimes}$

Energy, structure, and charge are fundamental quantities characterizing a molecule. Whereas the energy flow and structure change in chemical reactions are experimentally characterized, determining the atomic charges of a molecule in solution has been elusive, even for a triatomic molecule such as triiodide ion, $\mathrm{I}_{3}{ }^{-}$. Moreover, it remains to be answered how the charge distribution is coupled to the molecular geometry; which I-I bond, if two I-I bonds are unequal, dissociates depending on the electronic state. Here, femtosecond anisotropic $x$-ray solution scattering allows us to provide the following answers in addition to the overall rich structural dynamics. The analysis unravels that the negative charge of $\mathrm{I}_{3}{ }^{-}$is highly localized on the terminal iodine atom forming the longer bond with the central iodine atom, and the shorter I-I bond dissociates in the excited state, whereas the longer one in the ground state. We anticipate that this work may open a new avenue for studying the atomic charge distribution of molecules in solution and taking advantage of orientational information in anisotropic scattering data for solution-phase structural dynamics.

\footnotetext{
${ }^{1}$ Department of Chemistry and KI for the BioCentury, Korea Advanced Institute of Science and Technology (KAIST), Daejeon 34141, Republic of Korea. ${ }^{2}$ Center for Advanced Reaction Dynamics, Institute for Basic Science (IBS), Daejeon 34141, Republic of Korea. ${ }^{3}$ These authors contributed equally: Jun Heo, Jong Goo Kim. ${ }^{凶}$ email: hyotcherl.ihee@kaist.ac.kr
} 
F or understanding the reaction mechanism and reaction pathway of a chemical reaction, it is critical to track the change of the energy levels and three-dimensional structure in the reaction. Time-resolved spectroscopic techniques ${ }^{1-9}$ provide direct information on the energy flow based on the sensitivity to energy states, and time-resolved diffraction ${ }^{10-25}$ directly tracks the time-dependent structural change of a molecule based on the structural sensitivity. The charge distribution of a molecule or the flow of charge during a reaction plays a role as important as energy and structure, especially in reactions involving chargetransfer processes such as reactions in photoelectric and electrochemical devices ${ }^{26,27}$. Whereas the total charge or local charge distribution can be measured for molecules in solid or gas phases, no robust experimental method has yet been established to determine the atomic charges of individual atoms comprising a molecule in the liquid solution phase. For example, even for $\mathrm{I}_{3}{ }^{-}$, it has not yet been experimentally determined how the charge is distributed in each of the three $\mathrm{I}$ atoms in $\mathrm{I}_{3}{ }^{-}$in the ground state ${ }^{28-30}$ (Fig. 1a). Moreover, although it is known that $\mathrm{I}_{2}{ }^{-}$and I are formed by the excitation with $400 \mathrm{~nm}$ wavelength ${ }^{31}$, it is still elusive which specific bond dissociates among the two I-I bonds in $\mathrm{I}_{3}{ }^{-}$(Fig. 1b). It also remains to be determined which bond cleavage is responsible for the equilibrium between $\mathrm{I}_{3}{ }^{-}$and $\mathrm{I}^{-}+\mathrm{I}_{2}$ in the ground state.

In a TRXL experiment, the x-ray pulse incident after the laser pulse at the time delay, $\Delta t$, records the time evolution of the scattering patterns as well as the progress of the reaction and anisotropy. When a linearly polarized laser pulse interacts with an ensemble of molecules, the excitation probability of a molecule with a transition dipole, $\mu$, is proportional to $\cos ^{2} \phi$, where $\phi$ is the angle between the laser polarization $(\varepsilon)$ and the transition dipole $(\mu)$. As a result, the orientational distribution of the excited molecules is transiently anisotropic at the moment of laser excitation ${ }^{32}$. Such photoselective alignment is apparent in the experimental difference scattering patterns at early time delays (Supplementary Fig. 1). The experimental scattering patterns were decomposed into isotropic and anisotropic components by considering the orientational distribution of photoselectively aligned molecules, and the resulting isotropic difference curves $\left(\Delta S_{\text {iso }}\right)$ and anisotropic difference curves $\left(\Delta \mathrm{S}_{\text {aniso }}\right)$ were used for analysis to fully take advantage of the anisotropic information of the scattering patterns ${ }^{33}$. The $\Delta S_{a z}$ (azimuthally averaged difference curves), $\Delta S_{\text {iso }}$, and $\Delta S_{\text {aniso }}$ are shown in Supplementary Fig. 2.

To address these questions, we used time-resolved $\mathrm{x}$-ray liquidography (TRXL), also known as time-resolved $\mathrm{x}$-ray solution scattering, and analyzed the anisotropic scattering signal contained in the TRXL data. Here, the challenge is to properly consider the anisotropy in the data analysis. Recently, a few studies focusing on $\Delta \mathrm{S}_{\text {aniso }}$ beyond $\Delta \mathrm{S}_{\text {iso }}$ have been reported ${ }^{10,33}$, but only the anisotropy for the solute term was analyzed, while the anisotropy of the solute-solvent (cage) and solvent terms was not incorporated in the analysis due to their relatively small contributions to the scattering patterns. In general cases, the anisotropies of all three terms (solute, cage, and solvent) need to be analyzed ${ }^{34}$, and accordingly, a proper method to extract the additional information from the anisotropy in the scattering patterns is required. In this work, we present an advanced data analysis considering the anisotropy of all the contributing terms of the TRXL signal.

\section{Results and discussion}

Ground state structure and charge distribution. The TRXL data measured at the XFEL has a significantly better signal-to-noise ratio (SNR) than the previously reported curve measured at the synchrotron ${ }^{35}$. The improved SNR turned out critical for further refining the molecular geometry of the ground-state $\mathrm{I}_{3}{ }^{-}$and the atomic charge distribution, which could not be determined in the previous study. To determine the structure and charge distribution of $\mathrm{I}_{3}{ }^{-}$in the ground state, a structural fitting analysis was performed using $\Delta \mathrm{S}_{\mathrm{az}}$ at $100 \mathrm{ps}$. Since $\mathrm{I}_{2}{ }^{-}$and $\mathrm{I}$ are the only dominant species at $100 \mathrm{ps}$, the Debye scattering curves of $\mathrm{I}_{3}{ }^{-}$, $\mathrm{I}_{2}{ }^{-}$and I were considered for calculating the solute term in the structural fitting analysis (see Methods section and Supplementary Fig. 5 for details). We also checked the sensitivity of the different scattering curve on the structure and charge distribution of $\mathrm{I}_{3}{ }^{-}$by comparing cage terms calculated for several molecular geometries and charge distributions. As discussed in detail in Supplementary Information and Supplementary Fig. 4, the solute-solvent pair distribution function (PDF) is more sensitive to the atomic charge distribution than to the molecular geometry of $\mathrm{I}_{3}{ }^{-}$. The simulation results show that the charge distribution affects the solvent arrangement around the solute and accordingly a

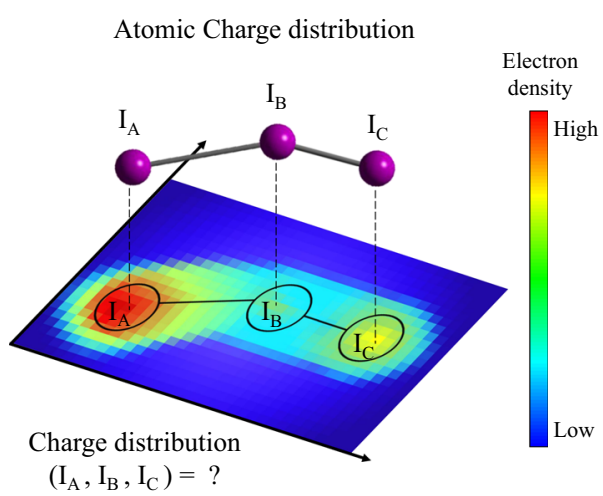

$\mathrm{b}$

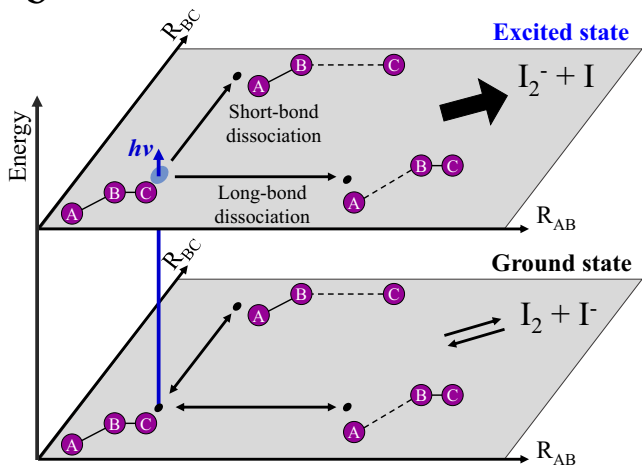

Fig. 1 Fundamental questions on dynamics and structure of $\mathbf{I}_{\mathbf{3}}-$. Simple yet fundamental questions on photodissociation dynamics of the triiodide ion in methanol such as $\mathbf{a}$ how the $1 \mathrm{e}^{-}$charge of the ion is distributed among three iodine atoms, how the charge distribution is related with the geometry, and b which I-I bond, the shorter $\left(R_{B C}\right)$ or the longer one $\left(R_{A B}\right)$, is broken upon dissociation in the excited and ground states remain to be answered. In $\mathbf{a}$, a case where the negative charge is localized on $\mathrm{I}_{\mathrm{A}}$ is represented as an example. In $\mathbf{b}$, two representative reaction pathways (short-bond dissociation and longbond dissociation) of the two-body dissociation of $\mathrm{I}_{3}{ }^{-}$are shown. See the main text for the details. In this work, time-resolved $\mathrm{x}$-ray liquidography with the femtosecond time resolution was used to address these questions. 
alters the cage term in the TRXL signal, demonstrating the possibility of determining the charge distribution from the TRXL data based on the change of the cage term. In this regard, a recent TRXL study provided evidence that the difference in charge distribution affects the cage orientation, which was tracked by the change of the cage term, and revealed the direction of charge transfer in a FeRu complex with the aid of molecular dynamics (MD) simulations and time-dependent density functional theory calculations ${ }^{36}$. Here, we aimed to determine the quantitative atomic charges of individual atoms comprising a molecule purely based on the TRXL data.

The structural fitting analysis was performed by optimizing structural parameters of $\mathrm{I}_{3}-$ (bond lengths of $\mathrm{I}_{\mathrm{A}}-\mathrm{I}_{\mathrm{B}}\left(\mathrm{R}_{\mathrm{AB}}\right)$ and $\mathrm{I}_{\mathrm{B}}-\mathrm{I}_{\mathrm{C}}\left(\mathrm{R}_{\mathrm{BC}}\right)$, and $\mathrm{I}_{\mathrm{A}}-\mathrm{I}_{\mathrm{B}}-\mathrm{I}_{\mathrm{C}}$ angle $(\theta)$ ) and $\mathrm{I}_{2}{ }^{-}$(the $\mathrm{I}-\mathrm{I}$ bond length $\left(\mathrm{R}\left(\mathrm{I}_{2}{ }^{-}\right)\right)$, where the three iodine atoms of $\mathrm{I}_{3}{ }^{-}$are labeled as $I_{A}, I_{B}$, and $I_{C}$, respectively. To consider the atomic charge distribution of $\mathrm{I}_{3}{ }^{-}$, new cage terms were generated by running MD simulations with $\mathrm{I}_{3}-$ that have various charge distributions. Because MD simulations are slow processes, they could not be simply implemented into a maximum likelihood fitting procedure. To cope with this situation, we conducted grid search procedures by generating grid points of all possible atomic charge distributions. We considered negative atomic charges ranging from 0 to $1 \mathrm{e}^{-}$and generated 66 grid points in total representing possible combinations of three atomic charges as shown in Fig. $2 \mathrm{a}$ and Supplementary Fig. 5. For each grid point, we performed the fitting iteratively until the solute structures to be optimized through the fitting process and those used to precalculate the cage term become self-consistent. The detailed procedure of the grid search is depicted in Supplementary Fig. 5. A plot of the weighted $\mathrm{R}$-factors (wRs) ${ }^{37,38}$ for all grid points (that is, a grid map) shown in Fig. 2a visualizes that the charge distribution of $\left(\mathrm{I}_{\mathrm{A}}, \mathrm{I}_{\mathrm{B}}\right.$, $\left.\mathrm{I}_{\mathrm{C}}\right)=(-0.9 \mathrm{e}, 0.0 \mathrm{e},-0.1 \mathrm{e})$ with an asymmetric bent structure $\left(\mathrm{R}_{\mathrm{AB}}=3.09 \pm 0.01 \AA, \mathrm{R}_{\mathrm{BC}}=2.96 \pm 0.01 \AA\right.$ and $\left.\theta=152 \pm 0.4^{\circ}\right)$ yielded the smallest $w R$. More details of the resulting fitting parameters are summarized in Supplementary Table 1. The optimized atomic charge distribution indicates that the negative charge is (i) asymmetrically located. The majority of the negative charge is localized on the terminal $\mathrm{I}$ atom $\left(\mathrm{I}_{\mathrm{A}}\right)$ forming the longer I-I bond $\left(\mathrm{I}_{\mathrm{A}}-\mathrm{I}_{\mathrm{B}}\right)$. We further inspected how the TRXL signal is altered depending on atomic charge distributions by comparing the fitting qualities at three representative grid points where the majority of the negative charge is (ii) equally distributed to $\mathrm{I}_{\mathrm{A}}$ and $\mathrm{I}_{\mathrm{C}}$, or (iii) localized on $\mathrm{I}_{\mathrm{B}}$ (Fig. 2d). The comparison confirms that the various charge distributions are distinguishable from the TRXL data, and the different charge distributions predominantly affect the TRXL signal in a small-angle region $\left(q<3 \AA^{-1}\right)$.

As can be seen in Fig. 2a, a local minimum point arises at around $\left(\mathrm{I}_{\mathrm{A}}, \mathrm{I}_{\mathrm{B}}, \mathrm{I}_{\mathrm{C}}\right)=(-0.3 \mathrm{e}, 0.0 \mathrm{e},-0.7 \mathrm{e})$, where the negative charge is localized on $\mathrm{I}_{\mathrm{C}}$ forming the shorter I-I bond. Similar to the global minimum point, the local minimum point has an asymmetric charge distribution where the negative charge is localized on one of the terminal I atoms. The fact that both global and local minima are found at asymmetric charge distributions reflects that the TRXL signal, which is sensitive to the symmetry of charge distribution, supports an asymmetric charge distribution. The global minimum of $\left(\mathrm{I}_{\mathrm{A}}, \mathrm{I}_{\mathrm{B}}, \mathrm{I}_{\mathrm{C}}\right)=(-0.9 \mathrm{e}, 0.0 \mathrm{e},-0.1 \mathrm{e})$ is more consistent with the consideration for the direction of bond cleavage, that is the shorter-bond dissociation vs the longerbond dissociation, as well as the results of ab initio MD simulations and x-ray photoelectron spectroscopy, as discussed later. The possibility that the local minimum accounts for the actual minor charge distribution is less probable although it cannot be ruled out.

We also checked the possibility of overfitting or model bias by randomly choosing $10 \%$ of the data points, fitting only the remaining $90 \%$ of the data, and checking the weighted $\mathrm{R}$-free factors $\left(w R_{\text {free }}\right)$. The $\mathrm{wR}_{\text {free }}$ is not significantly larger than the $\mathrm{w} R$, indicating that the obtained fit result is sound. Moreover, another evidence supporting this finding is provided by considering the rotational dynamics, which turn out to depend on the charge distribution (Fig. 2d), as discussed later. Nevertheless, we cannot rule out the possibility that the exact global minimum may change if different MD simulations to obtain the cage term coupled with the charge distribution are used instead of the current classical MD simulations. The exploration in this regard is beyond the scope of the current study, but the approach outlined in this work should provide a road for such efforts.

The charge distribution obtained from the TRXL data can be compared with those obtained from previous theoretical studies. For example, the previous works using CASSCF calculation and the MD simulation suggested that the symmetry breaking of $\mathrm{I}_{3}{ }^{-}$ occurs along with the charge localization toward the terminal iodine under methanol solvent condition due to the strong solvent-solute interaction involving hydrogen bonds, although those studies did not provide information on whether the negative charge is localized at the terminal iodine forming the shorter bond or the longer bond ${ }^{29,39}$. A later report using ab initio simulation results finally showed that the $\mathrm{I}_{3}{ }^{-}$in methanol has more negative charges on the terminal iodine forming the longer I-I bond ${ }^{30}$. Such charge localization toward the terminal iodine forming the longer bond was also reported for $\mathrm{I}_{3}{ }^{-}$in an aqueous solution by an ab initio MD simulation ${ }^{28}$, suggesting that hydrogen bonds induce the symmetry breaking and charge localization on the relatively loosely-bounded terminal iodine forming the longer bond. As a result, the atomic charge distribution extracted from the TRXL data is in excellent agreement with the previous theoretical expectations, and the hydrogen bond between the hydroxyl group of methanol and $\mathrm{I}_{3}{ }^{-}$ can account for the symmetry breaking and charge localization observed in this work. The agreement between the results obtained from the theoretical calculations and our experiment demonstrates that TRXL bodes well for the experimental determination of the atomic charge distribution of molecules in solution. Furthermore, the symmetry breaking features of $\mathrm{I}_{3}{ }^{-}$in terms of molecular geometry as well as the charge distribution obtained from TRXL are in line with those observed through Raman spectroscopy ${ }^{40}$ and $\mathrm{x}$-ray photoelectron spectroscopy ${ }^{28,30}$. Specifically, the Raman signal corresponding to the antisymmetric stretching mode, which has to be symmetry forbidden in the linear geometry, was observed for $\mathrm{I}_{3}{ }^{-}$in protic solvents such as water or ethanol while the signal is undetectable in acetonitrile, suggesting that $\mathrm{I}_{3}^{-}$is asymmetric in water or ethanol and symmetric in acetonitrile. Also, analysis of $\mathrm{I}(4 \mathrm{~d})$ core-level photoelectron spectra in conjunction with ab initio $\mathrm{MD}$ simulations support the symmetry breaking in I-I bond lengths with a more negative charge for the terminal iodine of the longer I-I bond while less negative charge for the terminal iodine of the shorter I-I bond, which is pronounced in water or methanol solvents ${ }^{30}$.

In addition to the charge distribution, the $\mathrm{I}_{3}{ }^{-}$structures in methanol from this work can be compared with those from DFT calculations and previous studies. The calculated structure using a DFT method, which is presented in Supplementary Information, is symmetric linear $\mathrm{I}_{3}{ }^{-}$with $2.95 \AA$ bond length, which is in stark contrast with the optimized asymmetric structure from our work, $2.96 \AA$ and $3.09 \AA$ for two I-I bond lengths with an I-I-I angle of $152.0^{\circ}$. On the other hand, Raman spectroscopy ${ }^{40}$ and $\mathrm{X}$-ray photoelectron spectroscopy ${ }^{30}$ provided evidence for asymmetric structure in protic solvents such as ethanol and water. Unlike the DFT structure, the structure using an ab initio MD simulation gives a good agreement with the TRXL structure ${ }^{30}$. Two I-I 

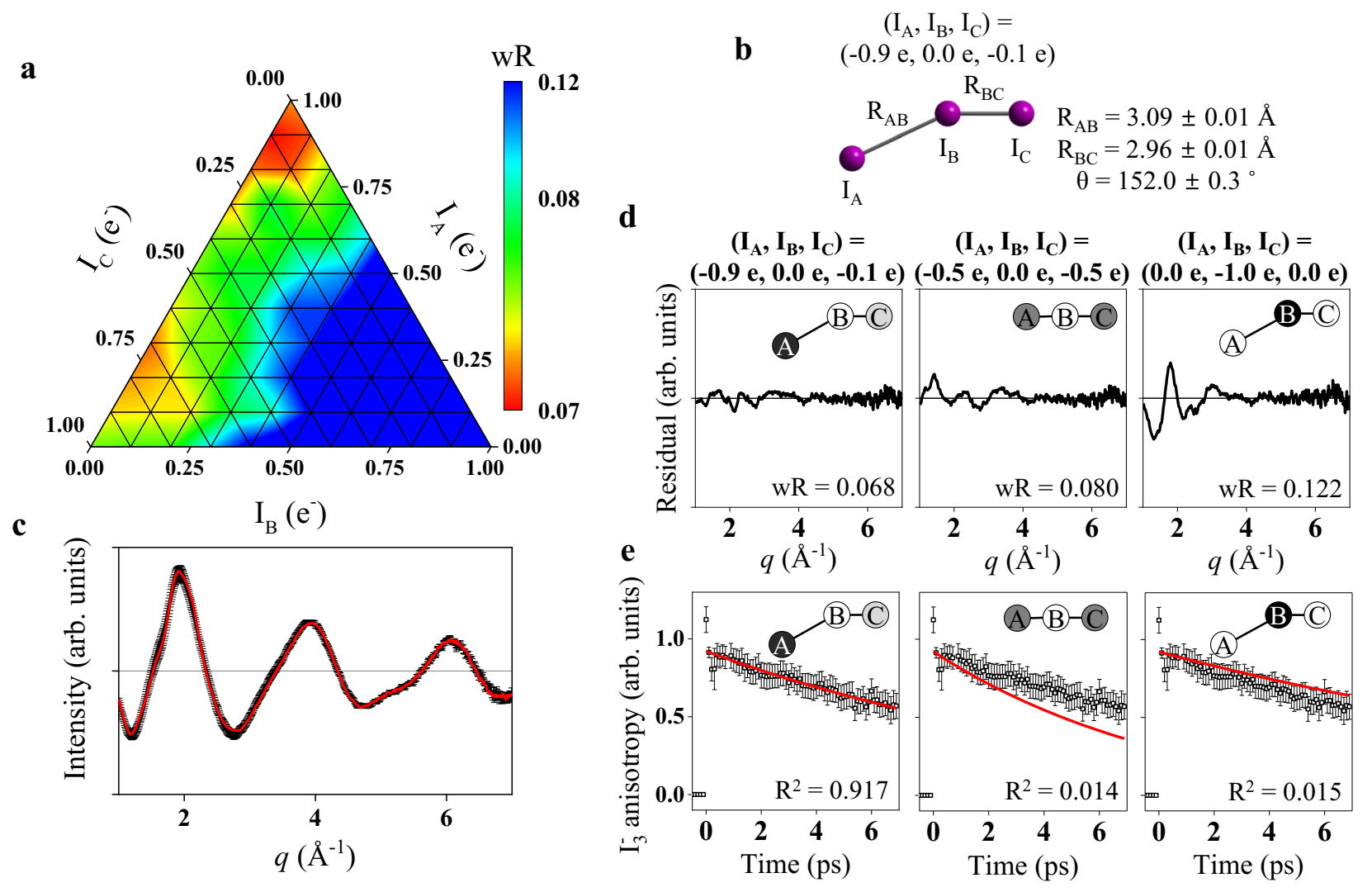

Fig. 2 Determination of the charge distribution in $\mathbf{I}_{\mathbf{3}}-$ in methanol. To determine the charge distribution, the data at 100 ps time delay was fitted using cage models calculated by MD simulations. The charge distribution of ground state $\mathrm{I}_{3}{ }^{-}$was analyzed by screening all possible charge distributions. a The weighted R-factors (wRs) are presented as a function of the charges of three I atoms. The best fit, which gives the smallest wR, was obtained with ( $I_{A}$, $I_{B}$, $\left.I_{C}\right)=(-0.9 e, 0.0 \mathrm{e},-0.1 \mathrm{e})$. A color scale represents the wRs of each charge model. $\mathbf{b}$ The optimized structure of $\mathrm{I}_{3}-$ in methanol solvent. $\mathbf{c}$ The difference curve at $100 \mathrm{ps}$ (black) with the corresponding standard error as error bars and the best fit curve (red) with $\left(I_{A}, I_{B}, I_{C}\right)=(-0.9$ e, 0.0 e, -0.1 e). d Residual differences between the experimental curve and the fit curve were obtained for three representative cases of charge distributions. The atomic charges and the corresponding wRs are also shown for all three cases. (Left) The best fit case, which gives the smallest residual. (Middle) The case with the negative charge equally distributed on both $\mathrm{I}_{\mathrm{A}}$ and $\mathrm{I}_{\mathrm{C}}$ as determined from DFT calculation. (Right) The case with the negative charge is localized on $\mathrm{I}_{\mathrm{B}}$. The magnitude of the atomic charge is schematically presented with white and black indicating zero and one full negative charge, respectively. The curves in c and $\mathbf{d}$ are on the same scale. e Comparison of the experimental anisotropy change (black dots with the corresponding standard deviations as error bars) and the calculated rotational correlation functions (RCFs) for the three representative cases of charge distributions shown in $\mathbf{d}$. The coefficient of determination $\left(R^{2}\right)$ for each case is also shown. The best agreement, which gives the $R^{2}$ value closest to 1 , is found with the first case, providing additional support for the determined charge distribution. Error bars were obtained by independently fitting the individual experimental data. Source data for panel c are provided as a Source Data file.

distances of the averaged structure of $\mathrm{I}_{3}{ }^{-}$in methanol from an ab initio MD simulation ( $2.94 \AA$ and $3.09 \AA$ ) agree well with those of the optimized structure $(2.96 \AA$ and $3.09 \AA)$ although the I-I-I angle from the ab initio MD simulation $\left(170.6^{\circ}\right)$ is noticeably larger than that from the our TRXL data $\left(152.0^{\circ}\right)$.

Determination of the direction of bond cleavage. Regarding the photodissociation process of $\mathrm{I}_{3}{ }^{-}$, two possibilities were considered: whether the shorter I-I bond $\left(\mathrm{I}_{\mathrm{B}}-\mathrm{I}_{\mathrm{C}}\right)$ or, the longer I-I bond $\left(\mathrm{I}_{\mathrm{A}}-\mathrm{I}_{\mathrm{B}}\right)$ dissociates. To elucidate which model is more consistent with the experimental data, we performed structural analysis by fitting $\Delta \mathrm{S}_{\text {iso }}$ and $\Delta \mathrm{S}_{\text {aniso }}$ simultaneously with each of the two models. In the fitting, time-dependent fitting parameters include $\mathrm{R}\left(\mathrm{I}_{2}{ }^{-}\right)$, the distance between the I radical and the center of $\mathrm{I}_{2}{ }^{-}\left(\mathrm{r}\left(\mathrm{I}_{-} \mathrm{I}_{2}^{-}\right)\right)$, the root-mean-square displacement of the Debye-Waller factor (DWF) for $r\left(\mathrm{I}_{-} \mathrm{I}_{2}^{-}\right)(\sigma)$, and other parameters to account for temperature change, optical Kerr response of solvent, the population of $\mathrm{I}_{2}^{-}$, and anisotropy (see "Structural analysis on $\Delta \mathrm{S}_{\text {iso }}$ and $\Delta \mathrm{S}_{\text {aniso }}(q, t>300 \mathrm{fs})$ " and "Structural analysis on $\Delta \mathrm{S}_{\text {iso }}$ and $\Delta \mathrm{S}_{\text {aniso }}(q, t \leq 300 \mathrm{fs})$ " in Supplementary
Information for details). We introduced a DWF $\left(\exp \left(-\sigma^{2} q^{2} / 2\right)\right)$ to $\mathrm{r}\left(\mathrm{I}_{-} \mathrm{I}_{2}{ }^{-}\right)$to describe the relatively free movement of the escaping I radical, yielding a broad distribution of the interatomic distances (see Methods section for details). Figure $3 \mathrm{~b}$ shows that at all time delays, the shorter-bond dissociation gives smaller wRs than the longer-bond dissociation, supporting that the shorter I-I bond of $\mathrm{I}_{3}-$ dissociates upon excitation. Then, we calculated the wRs for $\Delta \mathrm{S}_{\text {iso }}$ and $\Delta \mathrm{S}_{\text {aniso, }}$, separately (Figs. 3c, d), to examine which one between $\Delta \mathrm{S}_{\text {iso }}$ and $\Delta \mathrm{S}_{\text {aniso }}$ is mainly responsible for the resolving power. Since $\Delta S_{\text {iso }}$ contains the information on interatomic distances only without any directional information, $\Delta S_{\text {iso }}$ alone is not expected to distinguish well between the two scenarios well. In contrast to $\Delta \mathrm{S}_{\text {iso }}, \Delta \mathrm{S}_{\text {aniso }}$ is more likely to provide a clue to the direction of bond cleavage since the two scenarios among the dissociation of the shorter or longer bond should yield different orientational distributions of the excited molecules, which eventually make distinct anisotropic scattering patterns. As can be seen in Fig. 3c, the wRs of $\Delta S_{\text {iso }}$ are not significantly different for both models whereas in $\Delta S_{\text {aniso }}$ the shorter-bond dissociation gives smaller wRs than the longer-bond dissociation at all time delays, confirming the prediction. The possibility of determining 
a

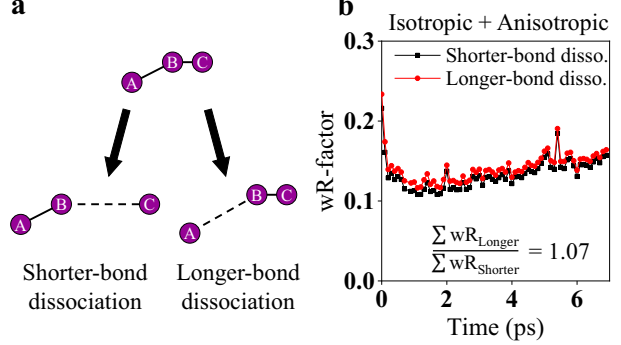

c

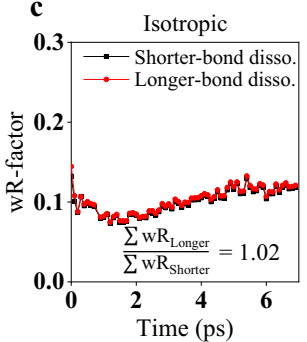

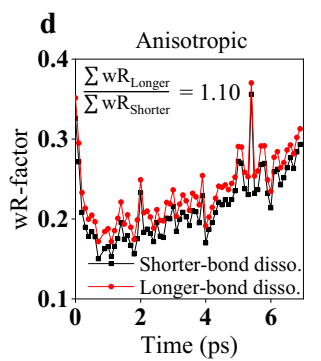

Fig. 3 Determination of the direction of bond dissociation in $\mathbf{I}_{\mathbf{3}}{ }^{-}$in methanol. The fs-TRXL data were fitted with two different dissociation models. a Two models for bond dissociation, the shorter-bond dissociation and the longer-bond dissociation, are shown schematically. $\mathbf{b}$ The wRs obtained when the shorter-bond dissociation (black) or the longer-bond dissociation (red) model is used to fit the isotropic and anisotropic data, simultaneously. c The wRs for the isotropic data. $\mathbf{d}$ The wRs for the anisotropic data. The smaller wRs with the shorter-bond dissociation at all time delays in the anisotropic data support the shorter-bond dissociation over the longer-bond dissociation, whereas such a distinction is relatively less clear in the isotropic data. In b-d, the ratio of the sum of wRs for the longer-bond dissociation and that of the shorter-bond dissociation is also shown. The larger ratio indicates the better fit quality for the shorter-bond dissociation model relative to the longer-bond dissociation.

the direction of bond cleavage based on the anisotropic scattering signal was further confirmed by simulations of difference scattering curves arising from two dissociation models (see Supplementary Information for details).

The shorter-bond dissociation model has been suggested by several theoretical studies. For example, a study using an ab initio calculation showed that the dissociation of the shorter I-I bond is favored in the fragmentation of $\mathrm{I}_{3}{ }^{-}$into $\mathrm{I}_{2}{ }^{-}$and $\mathrm{I}^{41}$. An MD simulation study also showed that the shift of the peak position in the low $q$ region of isotropic electron diffraction data is consistent with that of MD simulation with the assumption that the shorterbond dissociation model ${ }^{42}$. We note that the direction of bond cleavage could be determined solely based on the TRXL signal without the aid of ab initio calculations or MD simulations. Meanwhile, the ground-state PES from the ab initio calculation shows that the formation of $\mathrm{I}_{2}$ and $\mathrm{I}^{-}$is favored upon the dissociation of the longer I-I bond. The good agreement between TRXL data and the ab initio calculation for the excited state lends fidelity to the ab initio calculation for the ground state as well. If this is indeed the case, one can see that the bond dissociation and the charge distribution are coupled in such a way that the dissociation occurs in the direction where the redistribution of the atomic charge among atoms is minimized. In the ground state, where the formation of $\mathrm{I}_{2}$ and $\mathrm{I}^{-}$is operational in the equilibrium, the longer, weaker I-I bond is likely to be broken unlike in the excited state. The different bond dissociations in the excited and ground states and the determined atomic charge distribution provide insight into how the atomic charge distribution is linked to which bond dissociates.

Ultrafast structural dynamics of $\mathrm{I}_{3}{ }^{-}$. Besides the bond cleavage direction, the structural analysis on both $\Delta \mathrm{S}_{\text {iso }}$ and $\Delta \mathrm{S}_{\text {aniso, }}$, shown in Figs. 4a and 5a, respectively, unveils rich aspects of ultrafast structural dynamics such as the movement of the dissociating I and $\mathrm{I}_{2}{ }^{-}$, the escape dynamics of $\mathrm{I}$, the vibrational relaxation, and rotational diffusion dynamics. In Fig. $4 \mathrm{~b}, \mathrm{c}$, the time-dependent structural parameters, $r\left(\mathrm{I}_{-} \mathrm{I}_{2}^{-}\right), \sigma$ of the DWF for $\mathrm{r}\left(\mathrm{I}_{-} \mathrm{I}_{2}^{-}\right)$, and $\mathrm{R}\left(\mathrm{I}_{2}^{-}\right)$are shown.

As shown in Fig. $4 \mathrm{~b}, \mathrm{r}\left(\mathrm{I}^{-} \mathrm{I}_{2}{ }^{-}\right)$starts to increase right after the excitation and keeps increasing up to 7 ps, indicating that an I radical is formed by photoexcitation and the time-dependent $\mathrm{r}\left(\mathrm{I}-\mathrm{I}_{2}{ }^{-}\right)$represents the escape dynamics of I. Notably, the dynamics have two phases in terms of the escape speed of $\mathrm{I}$, as shown in Fig. 4b. The I radical moves away with the higher speed of $5.6 \pm 0.5 \AA / p s$ in the early time range ( $0 \mathrm{fs}<t<200 \mathrm{fs}$ ), and the speed decreases by a factor of 52 down to $0.11 \pm 0.01 \AA / p s$ in the late time range ( $200 \mathrm{fs}<t<6.9 \mathrm{ps})$. This observation infers that the newly born I radical exhibits relatively free movement since the motion occurs within the solvent cage in the early time range, and then the movement of the radical becomes a diffusionlimited process through other solvent molecules after the escape of the original solvent cage ${ }^{43}$. This initial escaping speed of I is compatible with the result obtained from the time-resolved electron diffraction method aided by MD simulations, by which the dissociation speed of $5.8 \AA$ $/ \mathrm{ps}$ was determined ${ }^{42}$. In a recent study of the photodynamics of $\mathrm{CH}_{2} \mathrm{I}_{2}$ studied by TRXL ${ }^{44}$, similar to $\mathrm{I}_{3}{ }^{-}$, the rapid dissociation upon photoexcitation of I radicals was observed. The reported escape speed of the dissociated I

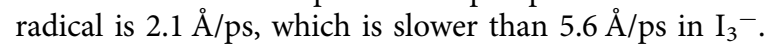

Similar to the time-dependent $r\left(\mathrm{I}_{2} \mathrm{I}_{2}{ }^{-}\right)$, the time-dependent $\sigma$ exhibit a two-phase rise in its temporal profile, as shown in Fig. $4 \mathrm{~b}$. In the early time range ( $0 \mathrm{fs}<t<200 \mathrm{fs}$ ), $\sigma$ is almost zero, meaning that $r\left(\mathrm{I}_{2} \mathrm{I}_{2}^{-}\right)$has a very narrow distribution in this time range. This observation can be attributed to the strong repulsive character of the excited-state PES of $\mathrm{I}_{3}{ }^{-}$along the axis of the shorter I-I bond $\left(\mathrm{R}_{\mathrm{BC}}\right)$ as reported in a previous ab initio calculation ${ }^{41}$ performed on asymmetric $\mathrm{I}_{3}{ }^{-}$. Accordingly, the wavepacket moving in the dissociative pathway leading to the formation of an I radical is expected to be highly coherent, resulting in a small $\sigma$. In the late time range $(200 \mathrm{fs}<t<6.9 \mathrm{ps}), \sigma$ increases up to $1 \AA$. As mentioned in the discussion on $\mathrm{r}\left(\mathrm{I}^{-} \mathrm{I}_{2}{ }^{-}\right)$, the movement of the I radical in this late time range corresponds to the diffusion process after colliding with the solvent cage. The collision to the solvent cage can result in the loss of coherency of the moving wavepacket and thus induce the increase of $\sigma$, corresponding to the broader distribution of $\mathrm{r}\left(\mathrm{I}^{-} \mathrm{I}_{2}^{-}\right)$.

The dynamics of $\mathrm{I}_{2}{ }^{-}$generated by the escape of the I radical are reflected in $\mathrm{R}\left(\mathrm{I}_{2}{ }^{-}\right)$(Fig. 4c). Similar to the time-dependent $\mathrm{r}\left(\mathrm{I}^{-} \mathrm{I}_{2}{ }^{-}\right), \mathrm{R}\left(\mathrm{I}_{2}{ }^{-}\right)$starts to increase right after the excitation. After $500 \mathrm{fs}, \mathrm{R}\left(\mathrm{I}_{2}{ }^{-}\right)$decreases until reaching the equilibrium distance $(3.25 \AA)$. The initial elongation and subsequent contraction of $\mathrm{R}\left(\mathrm{I}_{2}{ }^{-}\right)$occur with time constants of $0.19 \pm 0.01 \mathrm{ps}$ and $1.6 \pm 0.2 \mathrm{ps}$, respectively. The initial increase of $\mathrm{R}\left(\mathrm{I}_{2}{ }^{-}\right)$should arise from the initial motion of the vibrational wavepacket on the PES of $\mathrm{I}_{2}{ }^{-}$starting from the Franck-Condon (FC) region. Since the shorter $\mathrm{I}-\mathrm{I}$ bond $\left(\mathrm{I}_{\mathrm{B}}-\mathrm{I}_{\mathrm{C}}\right)$ is dissociated upon excitation, the remaining $\mathrm{I}_{A}-\mathrm{I}_{\mathrm{B}}$ converts into $\mathrm{I}_{2}{ }^{-}$in the excited state. Considering that $\mathrm{R}_{\mathrm{AB}}$ of $\mathrm{I}_{3}{ }^{-}$in the ground state (3.09 $\AA$ ) is shorter than the equilibrium $\mathrm{R}\left(\mathrm{I}_{2}{ }^{-}\right)(3.25 \AA)$, the wavepacket generated in the FC region should initially move in the direction of increasing $\mathrm{R}\left(\mathrm{I}_{2}{ }^{-}\right)$. Consistent with this expectation, $\mathrm{R}\left(\mathrm{I}_{2}{ }^{-}\right)$increases initially until $500 \mathrm{fs}$. The subsequent decrease of $\mathrm{R}\left(\mathrm{I}_{2}{ }^{-}\right)$can be assigned to the vibrational relaxation to low-lying vibrational levels of the 

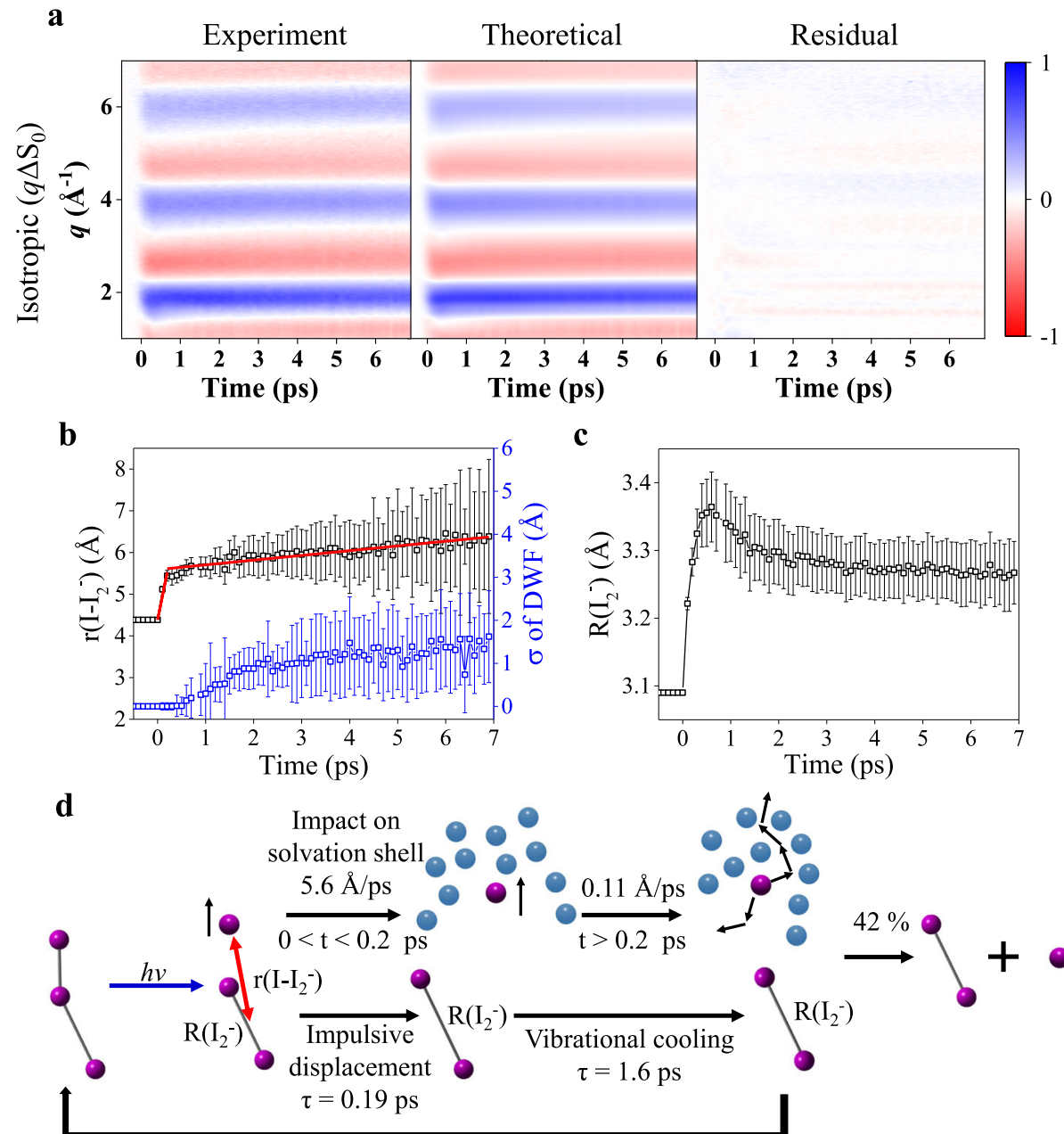

$$
\begin{gathered}
\text { Geminate recombination } \\
\tau_{1}=3.1 \mathrm{ps}, \tau_{2}=49 \mathrm{ps}, 58 \%
\end{gathered}
$$

Fig. 4 Structural dynamics from $\mathbf{q} \Delta \mathbf{S}_{\text {iso }}$ and the corresponding parameters and schematics on the dissociation dynamics of $\mathbf{I}_{\mathbf{3}}{ }^{-}$. The experimental $q \Delta S_{\text {iso }}$ curves are fitted, and the corresponding structural parameters are presented. a Experimental $q \Delta \mathrm{S}_{\text {iso }}$ (left), theoretical fits (middle), and the residual between the two (right) are presented (see Methods section for details). The individual curves and fits are also shown in Supplementary Fig. 14. All plots share a color scale representing the amplitude of the signal in absolute electronic units per solvent molecule. $\mathbf{b} r\left(I-I_{2}{ }^{-}\right)$and $\sigma$ of the DWF with the corresponding standard deviations as error bars are presented. The $r\left(\mathrm{I}_{-} \mathrm{I}_{2}^{-}\right)$and $\sigma$ were used as fitting parameters. The $\mathrm{r}\left(\mathrm{I}-\mathrm{I}_{2}{ }^{-}\right)$shows two-phase velocity change induced by the intermolecular potential between $\mathrm{I}_{2}-$ and I radical and friction between the solvent molecule and I radical. Red lines represent linear fits of $\mathrm{r}\left(\mathrm{I}_{-} \mathrm{I}_{2}^{-}\right)$in the two phases. c The $\mathrm{R}\left(\mathrm{I}_{2}{ }^{-}\right)$with the corresponding standard deviations as error bars are presented. $\mathbf{d}$ Schematics on the overall dynamics of $\mathrm{I}_{3}-$ photodissociation is shown. Upon $400 \mathrm{~nm}$ excitation, $\mathrm{I}_{3}{ }^{-}$dissociates into $\mathrm{I}_{2}{ }^{-}$and $\mathrm{I}$ radical. The dissociated $\mathrm{I}_{2}{ }^{-}$undergoes impulsive displacement and vibrational cooling with time constants of $0.19 \mathrm{ps}$ and $1.6 \mathrm{ps}$, respectively. The dissociated I radical moves away from $\mathrm{I}_{2}{ }^{-}$with two phases in terms of the escape speed of I ( 5.6 and $0.11 \AA / \mathrm{ps}$ in the early and late time ranges, respectively). $58 \%$ of the dissociated fragments undergo geminate recombination to form the $\mathrm{I}_{3}{ }^{-}$with two-time constants of $3.1 \mathrm{ps}$ and $49 \mathrm{ps}$. Source data for panel a are provided as a Source Data file.

anharmonic PES of $\mathrm{I}_{2}{ }^{-}$, leading to the decrease of averaged I-I distance. The time constant of 1.6 ps assigned to the vibrational relaxation of $\mathrm{I}_{2}^{-}$is in good agreement with the previously reported values determined from spectroscopy experiments ${ }^{43,45}$. In Fig. $4 \mathrm{~d}$, the overall reaction dynamics of $\mathrm{I}_{3}{ }^{-}$are summarized. In addition, the population dynamics of $\mathrm{I}_{2}{ }^{-}$investigated in a wider time window from -2 ps to 100 ps indicate the existence of the geminate recombination of $\mathrm{I}_{2}{ }^{-}$and $\mathrm{I}$ into $\mathrm{I}_{3}{ }^{-}$with two-time constants of $3.1 \pm 0.6$ ps and $49 \pm 22$ ps and the fraction of the geminate recombination of $58 \pm 11 \%$, as shown in Supplementary Fig. 10.

Rotational dephasing dynamics of $\mathrm{I}_{3}{ }^{-}$and $\mathrm{I}_{2}{ }^{-}$. Time-dependent anisotropy of $\mathrm{I}_{3}{ }^{-}$and $\mathrm{I}_{2}{ }^{-}$obtained from the structural analysis are shown in Fig. 5b, c, respectively. In Fig. 5b, time-dependent anisotropy of the hole of depleted $\mathrm{I}_{3}{ }^{-}$and the rotational diffusion dynamics corresponding to this anisotropy change are shown. The time-dependent anisotropy was fitted with a time constant of $13.5 \pm 7.9 \mathrm{ps}$, corresponding to the rotational diffusion process in which the $\cos ^{2} \phi$ orientational distribution of the ground-state hole relaxes to the isotropic distribution. In Fig. $5 c$, anisotropy of $\mathrm{I}_{2}-$ formed in the excited state and the corresponding rotational diffusion dynamics are shown. The change in the anisotropy of $\mathrm{I}_{2}^{-}$, also arises from the relaxation of the $\cos ^{2} \phi$ distribution to the isotropic orientational distribution of the excited-state molecules. Notably, unlike $\mathrm{I}_{3}{ }^{-}$, the temporal profile of anisotropy of $\mathrm{I}_{2}{ }^{-}$ exhibits two phases of the decay, including the rapid drop in the early time range $(0 \mathrm{ps}<t<1 \mathrm{ps})$ and the subsequent slow decrease in the late time range $(t>1 \mathrm{ps})^{45,46}$, which can be attributed to the 

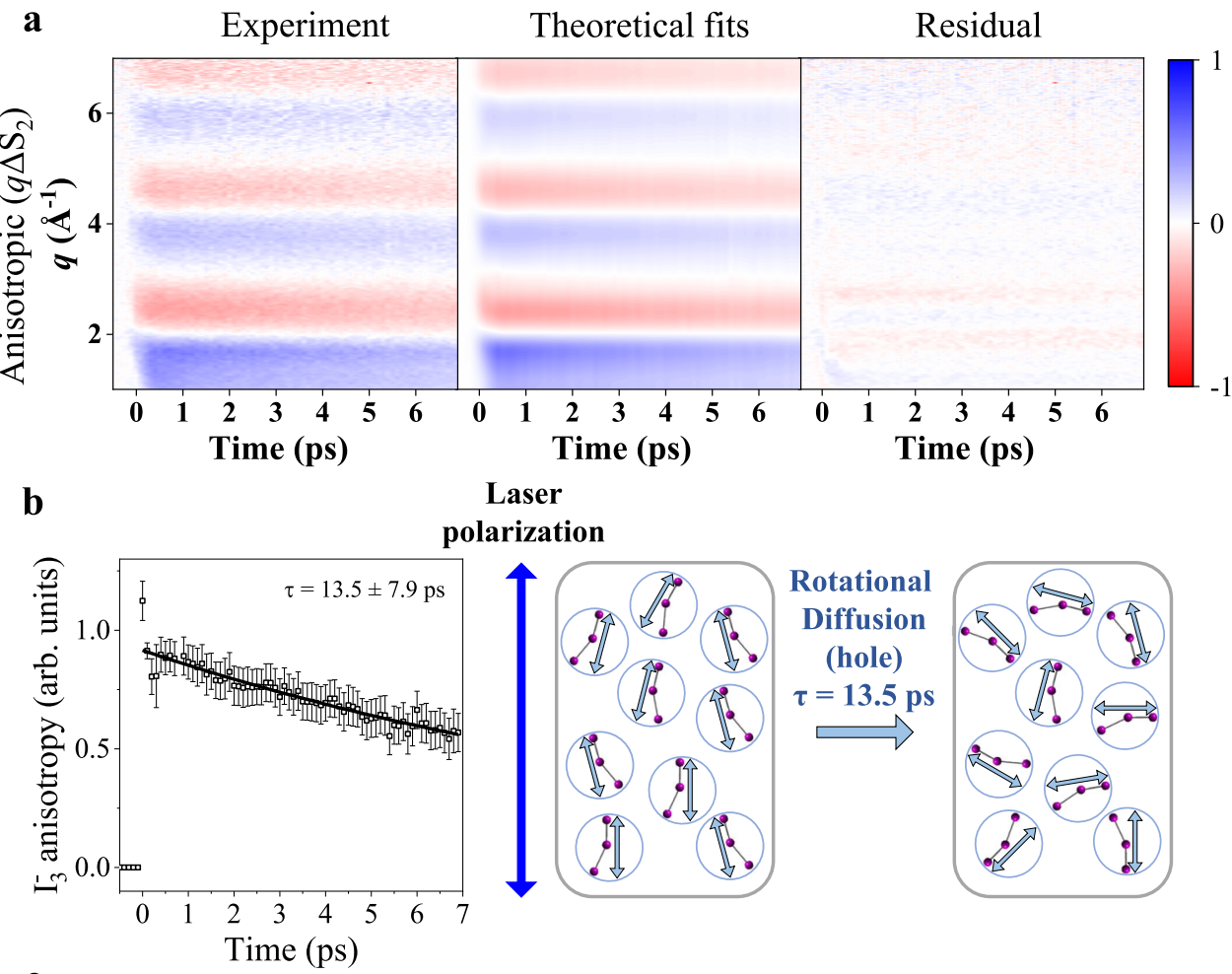

Laser
olarization
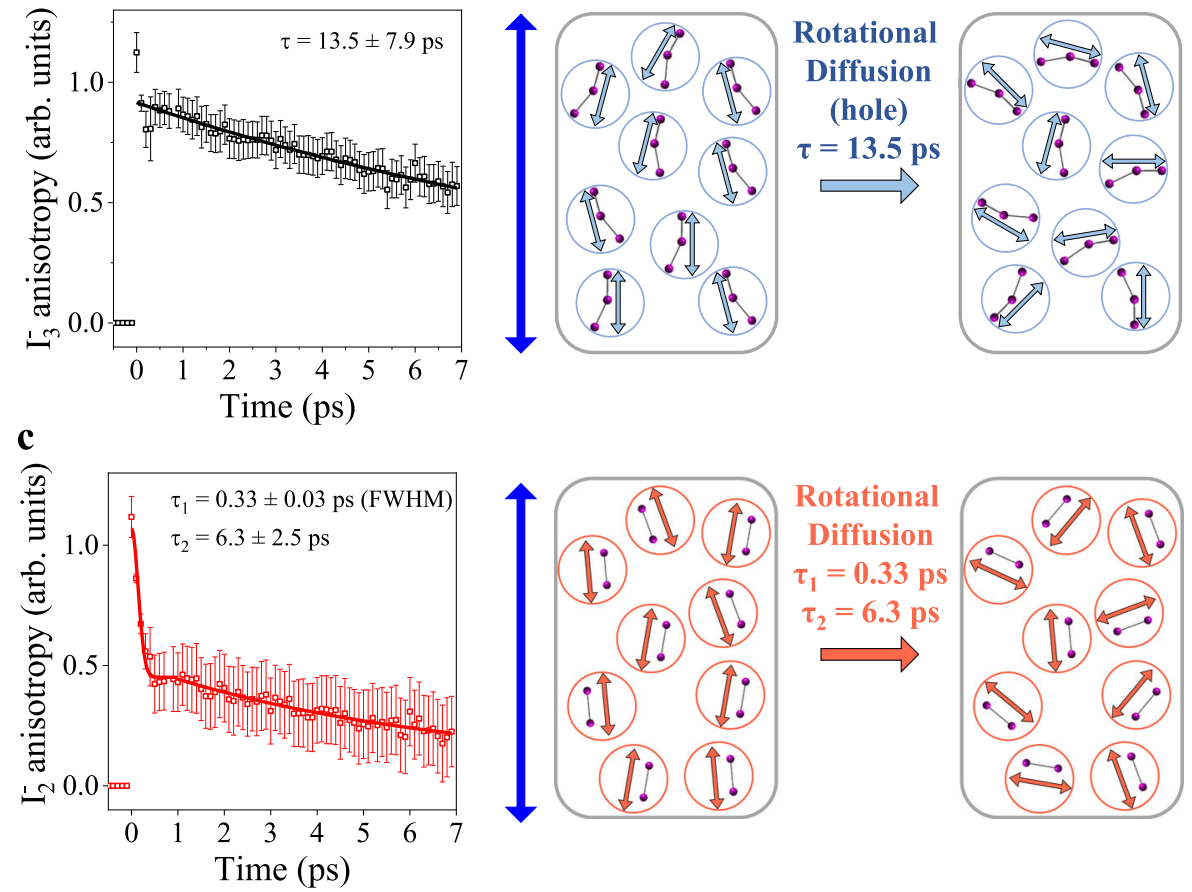

Fig. 5 Structural dynamics from $\mathbf{q} \Delta \mathbf{S}_{\text {aniso }}$ and the corresponding parameters and schematics on the rotational dynamics of $\mathbf{I}_{\mathbf{3}}{ }^{-}$and $\mathrm{I}_{\mathbf{2}}{ }^{-}$. $\mathbf{a}$ Experimentally acquired $q \Delta S_{\text {aniso }}$ (left), theoretical fits (middle), and the residual between the two (right) are presented (see Methods section for details). The individual curves and fits are also shown in Supplementary Fig. 14. All plots share a color scale representing the amplitude of the signal in absolute electronic units per solvent molecule. $\mathbf{b}$ The anisotropy coefficient of the $\mathrm{I}_{3}{ }^{-}$with the standard deviations as error bars and corresponding rotational dynamics are presented. The anisotropy of $\mathrm{I}_{3}{ }^{-}$originates from the holes of depleted $\mathrm{I}_{3}{ }^{-}$. This distribution is induced by a depleted portion of the $\mathrm{I}_{3}{ }^{-}$which is selectively excited along the polarization axis of the pump laser. c The anisotropy coefficient of the $\mathrm{I}_{2}{ }^{-}$with the standard deviations as error bars and corresponding rotational dynamics are presented. Because the dissociated $\mathrm{I}_{2}{ }^{-}$is rotationally excited by the dissociation, $\mathrm{I}_{2}{ }^{-}$shows the initial drop in the early time delay, which corresponds to the free rotor motion of the molecule. Source data for panel a are provided as a Source Data file.

initial free rotor motion and subsequent rotational diffusion dynamics of $\mathrm{I}_{2}{ }^{-}$. The initial free rotor motion in the first phase $(0 \mathrm{ps}<t<1 \mathrm{ps})$ can be fitted with a Gaussian function, yielding a similar full width at half maximum (FWHM) of $0.33 \pm 0.03$ ps to one in the previous study (Fig. $5 \mathrm{c})^{45}$. The anisotropy of $\mathrm{I}_{2}{ }^{-}$in the second phase $(t>1 \mathrm{ps})$ was fitted with an exponential function with a time constant of $6.3 \pm 2.5 \mathrm{ps}$, which corresponds to the rotational diffusion process of $\mathrm{I}_{2}{ }^{-}$. The two time constants $(13.5 \mathrm{ps}$ and $6.3 \mathrm{ps}$ ) observed in the TRXL data are consistent with $15 \mathrm{ps}$ and $5 \mathrm{ps}$ assigned to the rotational diffusion time constants of $\mathrm{I}_{3}^{-}$ and $\mathrm{I}_{2}{ }^{-}$, respectively, in a spectroscopic study ${ }^{46}$.

The rotational diffusion time constants of $\mathrm{I}_{3}{ }^{-}$and $\mathrm{I}_{2}{ }^{-}$ extracted from the experimental data were also compared with those from rotational correlation functions (RCFs) calculated using the MD snapshots used to calculate the cage terms ${ }^{47}$. The optimized structure $\left(\mathrm{R}_{\mathrm{AB}}=3.09 \AA, \mathrm{R}_{\mathrm{BC}}=2.96 \AA\right.$ and $\left.\theta=152^{\circ}\right)$ and charge distribution $\left(\left(\mathrm{I}_{\mathrm{A}}, \mathrm{I}_{\mathrm{B}}, \mathrm{I}_{\mathrm{C}}\right)=(-0.9 \mathrm{e}, 0.0 \mathrm{e},-0.1 \mathrm{e})\right)$ of $\mathrm{I}_{3}{ }^{-}$were used for this calculation. The resulting RCFs of $\mathrm{I}_{3}{ }^{-}$and $\mathrm{I}_{2}{ }^{-}$are shown in Supplementary Fig. 11. Both the RCFs of $\mathrm{I}_{3}{ }^{-}$ and $\mathrm{I}_{2}{ }^{-}$were fitted with an exponential function, yielding time constants of $15.8 \pm 0.2$ ps and $3.8 \pm 0.1 \mathrm{ps}$, respectively, showing good agreement with those extracted from the experimental data (13.5 ps for $\mathrm{I}_{3}^{-}$and 6.3 ps for $\mathrm{I}_{2}{ }^{-}$). It is noteworthy that RCFs change significantly depending on the charge distributions of $\mathrm{I}_{3}{ }^{-}$. For example, RCFs obtained for the cases where (ii) the charge is distributed equally at the two terminal $\mathrm{I}$ atoms $\left(\left(\mathrm{I}_{\mathrm{A}}, \mathrm{I}_{\mathrm{B}}\right.\right.$, $\left.\left.\mathrm{I}_{\mathrm{C}}\right)=(-0.5 \mathrm{e}, 0.0 \mathrm{e},-0.5 \mathrm{e})\right)$ and (iii) the charge is localized at the central $\mathrm{I}$ atom $\left(\left(\mathrm{I}_{\mathrm{A}}, \mathrm{I}_{\mathrm{B}}, \mathrm{I}_{\mathrm{C}}\right)=(0.0 \mathrm{e},-1.0 \mathrm{e}, 0.0 \mathrm{e})\right)$ decay with time constants of 8.9 ps and $22 \mathrm{ps}$, respectively (Supplementary Fig. 11c, d). Both cases where incorrect charge distributions were used for RCF calculations yielded the temporal profiles of the $\mathrm{RCF}_{\mathrm{S}}$ different from the experimental observations (Fig. 2e). 
Therefore, the fact that the $\mathrm{RCF}$ of $\mathrm{I}_{3}{ }^{-}$is consistent with the timedependent anisotropy of $\mathrm{I}_{3}{ }^{-}$only when the charge distribution of $\mathrm{I}_{3}{ }^{-}$is $\left(\mathrm{I}_{\mathrm{A}}, \mathrm{I}_{\mathrm{B}}, \mathrm{I}_{\mathrm{C}}\right)=(-0.9 \mathrm{e}, 0.0 \mathrm{e},-0.1 \mathrm{e})$ lends strong, additional support to the finding that this charge distribution determined from the structural analysis is reliable. These results demonstrate that the methodology using the cage term and proper treatment of the anisotropy in TRXL data analysis, presented in this work, offers experimental and analytics approach for determining the atomic charge distribution of a molecule in solution and the directionality of bond dissociation.

\section{Methods}

TRXL experiments and time-resolved difference curves. TRXL experiments were performed at the XSS beamline of PAL-XFEL (the Pohang Accelerator Laboratory $\mathrm{x}$-ray free-electron laser), using the experimental protocol in previous publications. The details are presented in the Supplementary Information. Twodimensional scattering images were averaged and decomposed to give isotropic and anisotropic one-dimensional scattering curves, $\mathrm{S}_{\text {iso }}(q, t)$ and $\mathrm{S}_{\text {aniso }}(q, t)$ respectively, as a function of momentum transfer, $q$, and time delay, $t$, between the laser and $\mathrm{x}$-ray pulses ${ }^{32}$. Time-resolved difference scattering curves, $\Delta S_{\text {iso }}(q, t)$ and $\Delta S_{\text {aniso }}(q, t)$, were generated by subtracting the reference data measured at $-20 \mathrm{ps}$ from the data at other time delays. Extracted $q \Delta \mathrm{S}_{\text {iso }}(q, t)$ and $q \Delta \mathrm{S}_{\text {aniso }}(q, t)$ are shown in Supplementary Figs. 2 and 14.

\section{Determining the molecular structure and atomic charge distribution of the} ground state. To determine the structure and charge distribution of $\mathrm{I}_{3}{ }^{-}$in the ground state, a structural fitting analysis was performed using $\Delta \mathrm{S}_{\mathrm{az}}$ at $100 \mathrm{ps}$. We considered Debye scattering curves of $\mathrm{I}_{3}{ }^{-}, \mathrm{I}_{2}{ }^{-}$, and I radical for calculating the solute term in the structural fitting analysis (see Fig. 1b). The cage term was calculated with the aid of MD simulations, and the solvent term was experimentally acquired in a separate experiment with a heating dye in methanol solution (see Supplementary Information and Supplementary Fig. 5 for details). We note that only theoretical $\Delta S_{\text {iso }}$ were considered for the structural fitting analysis since the contribution of $\Delta \mathrm{S}_{\text {aniso }}$ is negligible at $100 \mathrm{ps}$ as the rotational dephasing processes are completed before 100 ps, as can be seen in Supplementary Fig. 2.

The structural fitting analysis was performed by optimizing structural parameters of $\mathrm{I}_{3}{ }^{-}$(bond lengths of $\mathrm{I}_{\mathrm{A}}-\mathrm{I}_{\mathrm{B}}\left(\mathrm{R}_{\mathrm{AB}}\right)$ and $\mathrm{I}_{\mathrm{B}}-\mathrm{I}_{\mathrm{C}}\left(\mathrm{R}_{\mathrm{BC}}\right)$, and $\mathrm{I}_{\mathrm{A}}-\mathrm{I}_{\mathrm{B}}-\mathrm{I}_{\mathrm{C}}$ angle $(\theta)$ ) and $\mathrm{I}_{2}{ }^{-}$(the I-I bond length $\left(\mathrm{R}^{-} \mathrm{I}_{2}{ }^{-}\right)$), where the three iodine atoms of $\mathrm{I}_{3}{ }^{-}$are labeled as $\mathrm{I}_{\mathrm{A}}$, $I_{B}$, and $I_{C}$, respectively, as depicted in Fig. 1a. The cage terms were prepared before the structural fitting analysis for specific molecular geometries and charge distributions. Here, the basic strategy of the structural fitting analysis is to perform the analysis iteratively until the solute structures to be optimized through the fitting process, and the structures used to precalculate the cage term become self-consistent. Initially, a cage term of $\mathrm{I}_{3}{ }^{-}$was calculated by an $\mathrm{MD}$ simulation performed against the symmetric linear structure $\left(\mathrm{R}_{\mathrm{AB}}=\mathrm{R}_{\mathrm{BC}}=2.95 \AA, \theta=180^{\circ}\right)$ and symmetric charge distribution $\left(\left(\mathrm{I}_{\mathrm{A}}, \mathrm{I}_{\mathrm{B}}, \mathrm{I}_{\mathrm{C}}\right)=(-0.5 \mathrm{e}, 0 \mathrm{e},-0.5 \mathrm{e})\right)$, which is the optimized values from a density functional theory (DFT) calculation on $\mathrm{I}_{3}{ }^{-}$(see the section "Density functional theory calculation" in Methods section for the details on the DFT calculation). The cage term of $\mathrm{I}_{2}{ }^{-}$for the analysis was initially determined using the structure calculated from a DFT calculation $\left(\mathrm{R}\left(\mathrm{I}_{2}{ }^{-}\right)=3.23 \AA\right.$ ). Throughout the entire analysis, we used an atomic charge of $-0.5 \mathrm{e}$ for two iodine atoms of $\mathrm{I}_{2}{ }^{-}$since it is evident that the negative charge is equally distributed to the two iodine atoms. Then, the structural fitting analysis was performed using these cage terms by optimizing the structures of $\mathrm{I}_{3}{ }^{-}$and $\mathrm{I}_{2}{ }^{-}$. The structure of $\mathrm{I}_{3}{ }^{-}$resulting from the initial structural fitting has an asymmetric bent structure $\left(\mathrm{R}_{\mathrm{AB}}=3.14 \pm 0.01 \AA, \mathrm{R}_{\mathrm{BC}}=2.92 \pm 0.01 \AA, \theta=151.2 \pm 0.3^{\circ}\right)$. The structure of $\mathrm{I}_{2}{ }^{-}$was determined to be $\mathrm{R}\left(\mathrm{I}_{2}{ }^{-}\right)=3.28 \pm 0.01 \AA$. These resulting structures of $\mathrm{I}_{3}{ }^{-}$and $\mathrm{I}_{2}{ }^{-}$are not self-consistent with the structure used for the calculation of the cage term. Moreover, the asymmetric bent structure of $\mathrm{I}_{3}{ }^{-}$raises the possibility that the one quantum of the negative charge is asymmetrically distributed over three iodine atoms, unlike the symmetric linear structure, where the charge should be symmetrically distributed.

To consider the atomic charge distribution of $\mathrm{I}_{3}{ }^{-}$, new cage terms were generated by running $\mathrm{MD}$ simulations with the asymmetric bent $\mathrm{I}_{3}{ }^{-}$that have various charge distributions. Because MD simulations are slow processes, they could not be simply implemented into a maximum likelihood fitting procedure. To cope with this situation, we conducted grid search procedures by generating grid points of all possible atomic charge distributions. We considered negative atomic charges ranging from 0 to $1 \mathrm{e}^{-}$, so that we generated 66 grid points in total with a grid point representing a possible combination of three atomic charges as shown in Fig. 2a and Supplementary Fig. 5. For each grid point, which is a given charge distribution, we ran an MD simulation to obtain the corresponding cage term while the asymmetric bent structure of $\mathrm{I}_{3}{ }^{-}$determined from the initial structure fitting was used. Details on the MD simulations are described in the section "Molecular dynamics simulations" of Methods. Once all cage terms for all grid points were ready, we performed a fitting procedure for each grid point that refines the solute structures. Subsequently, we performed an additional optimization of the cage term and solute structures to attain self-consistency. Cage terms were calculated again using the refined structures of $\mathrm{I}_{3}^{-}$and $\mathrm{I}_{2}{ }^{-}$determined in the previous fitting procedure for all the grid points. Finally, using the refined cage terms, the solute structures were further optimized via structural fittings for the grid points. The detailed procedure of the grid search is depicted in Supplementary Fig. 5.

Structural analysis on $\Delta S_{\text {iso }}$ and $\Delta S_{\text {aniso }}(q, t>\mathbf{3 0 0} \mathrm{fs})$. In the structural analysis, the agreement between the theoretical and experimental difference scattering curves was quantified by the weighted R-factor (wR), which is given by the following equation.

$$
\mathrm{wR}(t)=\sqrt{\frac{\sum_{i} \frac{\left(\Delta S_{\text {theory,iso }}\left(q_{i}, t\right)-\Delta \mathrm{S}_{\text {iso }}\left(q_{i}, t\right)\right)^{2}}{\left(\sigma_{\text {iso }}\left(q_{i}, t\right)\right)^{2}}+\sum_{i} \frac{\left(\Delta \mathrm{S}_{\text {theory, aniso }}\left(q_{i}, t\right)-\Delta \mathrm{S}_{\text {aniso }}\left(q_{i}, t\right)\right)^{2}}{\left(\sigma_{\text {aniso }}\left(q_{i}, t\right)\right)^{2}}}{\sum_{i} \frac{\left(\Delta S_{\text {iso }}\left(q_{i}, t\right)\right)^{2}}{\left(\sigma_{\text {iso }}\left(q_{i}, t\right)\right)^{2}}+\sum_{i} \frac{\left(\Delta S_{\text {aniso }}\left(q_{i}, t\right)\right)^{2}}{\left(\sigma_{\text {aniso }}\left(q_{i}, t\right)\right)^{2}}}}
$$

Here, $\Delta S_{\text {iso }}$ and $\Delta \mathrm{S}_{\text {aniso }}$ are the experimentally measured isotropic and anisotropic difference scattering signals, respectively, $\Delta \mathrm{S}_{\text {theory,iso }}$ and $\Delta \mathrm{S}_{\text {theory,aniso }}$ are the theoretical difference scattering curves calculated during the fitting processes, and $\sigma_{\text {iso }}$ and $\sigma_{\text {aniso }}$ are the standard errors of $\Delta \mathrm{S}_{\text {iso }}$ and $\Delta \mathrm{S}_{\text {aniso }}$, respectively. The minimization of the $\mathrm{wR}$ was performed to refine the molecular structure using the MINUIT package written at CERN and the error analysis was performed by MINOS, a built-in algorithm in the MINUIT software ${ }^{48}$. Structural and anisotropic parameters were optimized by minimizing the $\mathrm{wR}$. The structure of ground state $\mathrm{I}_{3}{ }^{-}$was fixed to that determined from the analysis of the 100-ps data. The fitting was conducted for each time delay by constructing the theoretical scattering curves calculated using the following equations.

$$
\begin{aligned}
\Delta \mathrm{S}_{\text {theory,iso }}(q, t)= & \mathrm{B}_{0}(t) *\left(\mathrm{~S}_{\mathrm{e}, \text { iso }}(q, t)-\mathrm{S}_{\mathrm{g}, \text { iso }}(q, t)\right)+\left.\mathrm{B}_{1}(t) * \frac{\partial S(q)}{\partial T}\right|_{\rho} \\
\Delta \mathrm{S}_{\text {theory,aniso }}(q, t)= & \mathrm{B}_{0}(t) *\left(\mathrm{~A}_{\mathrm{e}}(t) * \mathrm{~S}_{\mathrm{e}, \text { aniso }}(q, t)-\mathrm{A}_{\mathrm{g}}(t) * \mathrm{~S}_{\mathrm{g}, \text { aniso }}(q, t)\right) \\
& +\mathrm{B}_{2}(t) * \Delta \mathrm{S}_{\text {Kerr }}
\end{aligned}
$$

In these equations, $S_{e, \text { iso }}$ and $S_{g, \text { iso }}$ are the theoretical isotropic scattering curves of the products $\left(\mathrm{I}_{2}{ }^{-}\right.$and $\left.\mathrm{I}\right)$ and the reactant $\left(\mathrm{I}_{3}{ }^{-}\right)$, respectively. $\mathrm{S}_{\mathrm{e} \text {,aniso }}$ and $\mathrm{S}_{\mathrm{g} \text {,aniso }}$ are the theoretical anisotropic scattering curves of $\mathrm{I}_{2}{ }^{-}$and $\mathrm{I}_{3}{ }^{-}$. These scattering curves, $\mathrm{S}_{\mathrm{e}, \mathrm{iso}}, \mathrm{S}_{\mathrm{g}, \mathrm{iso}}, \mathrm{S}_{\mathrm{e}, \text { aniso, }}$ and $\mathrm{S}_{\mathrm{g} \text {,aniso }}$ are composed of the solute term and the cage term. $\left.\frac{\partial S(q)}{\partial T}\right|_{\rho}$ is the contribution of the solvent heating, which is measured in separate experiments using a heating dye $\mathrm{e}^{19,49,50} . \Delta \mathrm{S}_{\mathrm{Kerr}}$ is the anisotropic difference scattering signal arising from the Kerr effect of solvent molecules. $\left.\frac{\partial S(q)}{\partial T}\right|_{\rho}$ and $\Delta S_{\text {Kerr }}$, used for the structural analysis, are shown in Supplementary Fig. 13. $\Delta S_{\text {Kerr }}$ was extracted from the anisotropic solvent heating data, which was obtained from a separate TRXL experiment on a heating dye (see the "Structural analysis on $\Delta \mathrm{S}_{\text {iso }}$ and $\Delta \mathrm{S}_{\text {aniso }}(q, t>300 \mathrm{fs})$ " section of Supplementary Information for the details). $\mathrm{B}_{0}(t), \mathrm{B}_{1}(t)$ and $\mathrm{B}_{2}(t)$ reflect the population dynamics of $\mathrm{I}_{2}^{-}$, and the intensity of the solvent heating signal, and the intensity of the solvent Kerr signal, respectively. The fitted results are presented in Figs. 4 and 5. The detailed procedure and equations to calculate $\mathrm{S}_{\mathrm{e} \text {,iso }}$ and $\mathrm{S}_{\text {g,iso }}$ in Eq. (2) and $\mathrm{S}_{\mathrm{e} \text {,aniso }}$ and $\mathrm{S}_{\mathrm{g}, \text { aniso }}$ in Eq. (3) are described in the section "Structural analysis on $\Delta \mathrm{S}_{\text {iso }}$ and $\Delta \mathrm{S}_{\text {aniso }}(q, t>300 \mathrm{fs}$ )" of Supplementary Information.

We constructed the theoretical isotropic and anisotropic scattering curves following Eqs. (2) and (3), and minimized the discrepancy between the theoretical and experimental data. To do so, we minimized the wR given by Eq. (1), by optimizing several structural parameters. In detail, the I-I bond length $\left(\mathrm{R}\left(\mathrm{I}_{2}{ }^{-}\right)\right)$, the distance between the I radical and the center of $\mathrm{I}_{2}^{-}\left(\mathrm{r}\left(\mathrm{I}-\mathrm{I}_{2}-\right)\right)$, the root-meansquare displacement of the Debye-Waller factor (DWF) for $\mathrm{r}\left(\mathrm{I}-\mathrm{I}_{2}{ }^{-}\right)(\sigma)$ were used as fitting parameters. Here, the DWF $\left(\exp \left(-\sigma^{2} q^{2} / 2\right)\right)$ was introduced to describe the relatively free movement of the escaping I radical, yielding a broad distribution of the interatomic distance between the I radical and the center of $\mathrm{I}_{2}{ }^{-}$(see the "Structural analysis on $\Delta \mathrm{S}_{\text {iso }}$ and $\Delta \mathrm{S}_{\text {aniso }}(q, t>300 \mathrm{fs})$ " section of Supplementary Information). Here, we note that $\mathrm{R}\left(\mathrm{I}_{2}^{-}\right), \mathrm{r}\left(\mathrm{I}-\mathrm{I}_{2}^{-}\right), \sigma, \mathrm{A}_{\mathrm{e}}(t), \mathrm{A}_{\mathrm{g}}(t), \mathrm{B}_{0}(t), \mathrm{B}_{1}(t)$, and $\mathrm{B}_{2}(t)$ were used as the time-dependent fitting parameters. Among these parameters, $\mathrm{R}\left(\mathrm{I}_{2}{ }^{-}\right), \mathrm{r}\left(\mathrm{I}_{-} \mathrm{I}_{2}{ }^{-}\right)$and $\mathrm{B}_{0}(t)$ were used for both isotropic and anisotropic fitting processes, $\sigma$ and $B_{1}(t)$ were used for the isotropic curve fitting, and the others, $\mathrm{A}_{\mathrm{e}}(t), \mathrm{A}_{\mathrm{g}}(t)$, and $\mathrm{B}_{2}(t)$ were used for the anisotropic data.

Structural analysis on $\Delta S_{\text {iso }}$ and $\Delta S_{\text {aniso }}(q, t \leq \mathbf{3 0 0}$ fs). To obtain the transient structures at time delays shorter than the temporal width of the experimental IRF $(t \leq 300 \mathrm{fs})$, we performed a structural analysis considering the convolution of the molecular response with the IRF. Following the previously reported procedures ${ }^{11,23}$, an approach to fit the experimental curves within the experimental IRF ( $t \leq 300 \mathrm{fs}$ ) was slightly modified from that used for the time delays larger than the experimental IRF, described in the section "Structural analysis on $\Delta \mathrm{S}_{\text {iso }}$ and $\Delta \mathrm{S}_{\text {aniso }}(q, t>300 \mathrm{fs})$ " of Methods. In detail, the time-dependent parameters for the structures of $\mathrm{I}_{2}{ }^{-}$and the dissociating I radical, $\sigma$ of the DWF, anisotropy coefficients $\left(\mathrm{A}_{\mathrm{e}}(t)\right.$, and $\left.\mathrm{A}_{\mathrm{g}}(t)\right)$ and the population of $\mathrm{I}_{2}{ }^{-}\left(\mathrm{B}_{0}(t)\right)$ were modeled by a quartic polynomial function as follows:

$$
\mathrm{x}(\mathrm{t})=\sum_{k=0}^{4} a_{4-k} t^{4-k}
$$


where $\mathrm{a}_{4-k}$ is the coefficient of the polynomial function. The isotropic and anisotropic scattering curves for the solute signal in $S_{\text {inst.iso }}(q, t)$ and $S_{\text {inst.aniso }}(q, t)$ were calculated based on $x(t)$ from which the molecular structures were constructed and the DWF for $\mathrm{r}\left(\mathrm{I}-\mathrm{I}_{2}{ }^{-}\right)$was calculated. Subsequently, the instantaneous theoretical difference scattering curves, $\Delta \mathrm{S}_{\text {inst }}(q, t)$, were calculated using the following equation:

$$
\begin{gathered}
\Delta \mathrm{S}_{\text {inst, iso }}(q, t)=\mathrm{B}_{0}(t) *\left[\mathrm{~S}_{\mathrm{e}, \text { iso }}(q, t)-\mathrm{S}_{\mathrm{g}, \text { iso }}(q)\right]+\left.\mathrm{B}_{1}(t) * \frac{\partial S(q)}{\partial T}\right|_{\rho} \\
\Delta \mathrm{S}_{\text {inst,aniso }}(q, t)=\mathrm{B}_{0}(t) *\left[\mathrm{~S}_{\mathrm{e}, \text { aniso }}(q, t) \mathrm{A}_{\mathrm{e}}(t)-\mathrm{S}_{\mathrm{g}, \text { aniso }}(q) \mathrm{A}_{\mathrm{g}}(t)\right]+\mathrm{B}_{2}(t) * \Delta \mathrm{S}_{\text {Kerr }}
\end{gathered}
$$

where the terms in Eqs. (5) and (6) are the same as those in Eqs. (2) and (3). The fraction of the heating $\left(\mathrm{B}_{1}(t)\right)$ and Kerr $\left(\mathrm{B}_{2}(t)\right)$ signals was determined by using the SANOD during the fitting process minimizing the residual of the fit ${ }^{51} \cdot \mathrm{B}_{0}(t), \mathrm{A}_{\mathrm{e}}(t)$, and $\mathrm{A}_{\mathrm{g}}(t)$ were also modeled by quadratic polynomial functions and the coefficients of the polynomial functions were used as fitting parameters. Then, $\Delta S_{\text {inst,iso }}(q, t)$ and $\Delta S_{\text {inst,aniso }}(q, t)$ were convoluted by the experimental $\operatorname{IRF} \operatorname{IRF}(t)$, by the following equations.

$$
\begin{gathered}
\Delta \mathrm{S}_{\text {theory,iso }}(q, t \leq 300 f s)=\Delta \mathrm{S}_{\text {inst,iso }}(q, t) \bigotimes \operatorname{IRF}(t) \\
\Delta \mathrm{S}_{\text {theory,aniso }}(q, t \leq 300 f s)=\Delta \mathrm{S}_{\text {inst,aniso }}(q, t) \bigotimes \operatorname{IRF}(t)
\end{gathered}
$$

Under the constraints where the polynomial functions smoothly connect the structure at $0 \mathrm{fs}$, which is the structure of the $\mathrm{I}_{2}{ }^{-}$at Frank-Condon region, and the structures at $300 \mathrm{fs}$ determined following the procedures described in "Structural analysis on $\Delta \mathrm{S}_{\text {iso }}$ and $\Delta S_{\text {aniso }}(q, t>300 \mathrm{fs})$ " of Methods section, the coefficients of the polynomial functions for each parameter were optimized by minimizing the wR, which is given by the following equation:

$$
\mathrm{wR}=\sqrt{\frac{\sum_{j=\text { time delay }} \sum_{i}\left[\frac{\left(\Delta S_{\text {theory iso }}\left(q_{i}, t_{j}\right)-\Delta S_{\text {sion }}\left(q_{i}, t_{j}\right)\right)^{2}}{\left(\sigma_{\text {iso }}\left(q_{i}, t_{j}\right)\right)^{2}}+\frac{\left(\Delta S_{\text {theory, aniso }}\left(q_{i}, t_{j}\right)-\Delta S_{\text {anio }}\left(q_{i}, t_{j}\right)\right)^{2}}{\left(\sigma_{\text {aniso }}\left(q_{i}, t_{j}\right)\right)^{2}}\right]}{\sum_{j=\text { time delay }} \sum_{i}\left[\frac{\left(\Delta S_{\text {iso }}\left(q_{i}, t_{j}\right)\right)^{2}}{\left(\sigma_{\text {iso }}\left(q_{i}, t_{j}\right)\right)^{2}}+\frac{\left(\Delta S_{\text {aniso }}\left(q_{i}, t_{j}\right)\right)^{2}}{\left.\left(\sigma_{\text {anis }}\left(q_{i}, t_{j}\right)\right)^{2}\right]}\right.}}
$$

where $\sigma_{\text {iso }}$ and $\sigma_{\text {aniso }}$ is the standard deviation of the isotropic and anisotropic difference scattering intensity at each $q$ and $t$. Finally, the resultant $\Delta S_{\text {theory,iso }}(q, t \leq 300 \mathrm{fs})$ and $\Delta \mathrm{S}_{\text {theory,aniso }}(q, t \leq 300 \mathrm{fs})$ were concatenated with $\Delta \mathrm{S}_{\text {theory,iso }}(q, t>300 \mathrm{fs})$ and $\Delta \mathrm{S}_{\text {theory,aniso }}(q, t>300 \mathrm{fs})$, respectably, giving rise to $\Delta \mathrm{S}_{\text {theory,iso }}$ and $\Delta \mathrm{S}_{\text {theory,aniso }}$ shown in Figs. $4 \mathrm{a}$ and $5 \mathrm{a}$, respectively. The optimized $x(t)$ values were concatenated with the corresponding parameters used to calculate the solute signal of $\Delta \mathrm{S}_{\text {theory,iso }}(q, t>300 \mathrm{fs})$ and $\Delta S_{\text {theory.aniso }}(q, t>300 \mathrm{fs})$ and are represented in Figs. $4 b, c$ and $5 b$, c. See the section "Structural analysis on $\Delta \mathrm{S}_{\text {iso }}$ and $\Delta \mathrm{S}_{\text {aniso }}(q, t \leq 300 \mathrm{fs})$ " of Supplementary Information for more details.

Anisotropic cage term. The change of solute structure is accompanied by the change of cage structure around the solute molecule. We have developed a methodology to calculate the anisotropic cage term for any desired distribution of the solute molecules. First, we defined unit angular vectors $\left(\overrightarrow{v_{m}}\right)$, which point evenly spaced spherical coordinates on the unit sphere using the spiral methods ${ }^{52}$, as shown in Supplementary Fig. 6a. A unit angular vector indicates the direction of the corresponding orientation-dependent PDF, $g_{j k}\left(r, \overrightarrow{v_{m}}\right)$, to construct. To calculate $g_{j k}\left(r, \overrightarrow{v_{m}}\right)$, we identified the desired atoms (for example, $k$ ) of solvent molecules within the polyhedral cone of the expanded sphere and calculated the PDF for the cross pair of an atom of a solute molecule (for example, $j$ ) and the selected solvent atoms within each polyhedral cone specified by $\overrightarrow{v_{m}}$. The cage signal corresponding to the orientation-dependent PDF is given by the following equation.

$$
\mathrm{S}_{\text {cage }}\left(\overrightarrow{\boldsymbol{q}}, \overrightarrow{\boldsymbol{v}_{\boldsymbol{m}}}\right)=2 * \sum_{j} \sum_{k} f_{j}(|\overrightarrow{\boldsymbol{q}}|) f_{k}(|\overrightarrow{\boldsymbol{q}}|) n_{k} \int \frac{4 \pi r^{2}}{N}\left(g_{j k}\left(r, \overrightarrow{\boldsymbol{v}_{\boldsymbol{m}}}\right)-1\right) \cos \left(r \overrightarrow{\boldsymbol{q}} \cdot \overrightarrow{\boldsymbol{v}_{\boldsymbol{m}}}\right) d r
$$

Then, the cage signal for a single solute molecule embedded in solvent molecules is calculated by summing all $\mathrm{S}_{\text {cage }}\left(\overrightarrow{\boldsymbol{q}}, \overrightarrow{\boldsymbol{v}_{m}}\right)$, as follows.

$$
\begin{aligned}
\mathrm{S}_{\text {cage }}(\overrightarrow{\boldsymbol{q}}) & =\sum_{m=1}^{N} \mathrm{~S}_{\text {cage }}\left(\overrightarrow{\boldsymbol{q}}, \overrightarrow{\boldsymbol{v}_{\boldsymbol{m}}}\right) \\
& =2 * \sum_{m}^{N} \sum_{j} \sum_{k} f_{j}(|\overrightarrow{\boldsymbol{q}}|) f_{k}(|\overrightarrow{\boldsymbol{q}}|) n_{k} \int \frac{4 \pi r^{2}}{N}\left(g_{j k}\left(r, \overrightarrow{\boldsymbol{v}_{\boldsymbol{m}}}\right)-1\right) \cos \left(r \overrightarrow{\boldsymbol{q}} \cdot \overrightarrow{\boldsymbol{v}_{\boldsymbol{m}}}\right) d r
\end{aligned}
$$

Using Eq. (11), we calculated the scattering pattern from the solute-solvent pair for a solute molecule with any orientation. Generally, a single MD snapshot cannot give a satisfactory cage signal due to the insufficient sampling of the positions and orientations of solvent molecules. To compensate for this issue, we calculated the averaged orientation-dependent PDFs extracted from the 200,000 MD snapshots as follows:

$$
g_{j k}^{a v e}\left(r, \overrightarrow{v_{m}}\right)=\sum_{i=1}^{200,000} g_{j k}^{(i)}\left(r, \overrightarrow{v_{m}}\right)
$$

where $g_{j k}^{(i)}\left(r, \overrightarrow{v_{m}}\right)$ is the orientation-dependent PDF calculated from the $i$-th MD snapshot and $g_{j k}^{a v e}\left(r, \overrightarrow{v_{m}}\right)$ is the averaged orientation-dependent PDFs over 200,000 MD snapshots (see Supplementary Fig. 7c). Using the averaged orientationdependent PDFs $\left(g_{j k}^{\text {ave }}\left(r, \overrightarrow{\boldsymbol{v}_{m}}\right)\right)$, the cage scattering pattern arising from the anisotropic orientation of the solute molecules was calculated as follows.

$$
\mathrm{S}_{\text {cage }}(\overrightarrow{\boldsymbol{q}})=\sum_{m}^{N} \sum_{j} \sum_{k} f_{j}(|\overrightarrow{\boldsymbol{q}}|) f_{k}(|\overrightarrow{\boldsymbol{q}}|) n_{k} \int \frac{4 \pi r^{2}}{N}\left(g_{j k}^{\text {ave }}\left(r, \overrightarrow{\boldsymbol{v}_{m}}\right)-1\right) \cos \left(r \overrightarrow{\boldsymbol{q}} \cdot \overrightarrow{\boldsymbol{v}_{m}}\right) d r
$$

So far, we explained how the cage scattering term for a single solute molecule with a certain fixed orientation embedded in a box of solvent molecules. The next step is to consider the effect of the anisotropic distribution induced by the photoselective alignment. The anisotropic distribution of excited molecules induced by a linearly polarized laser pulse is given by $\mathrm{P}(\phi) \propto \cos ^{2} \phi$, where $\phi$ stands for the angle between the laser polarization and the transition dipole moment ${ }^{32}$. To consider the effect of molecular orientations, we rotated each MD snapshot to retrieve the molecular orientations induced by the photoexcitation where the rotated solute molecules have the $\cos ^{2}$ distribution, as shown in Supplementary Fig. 7b. For each of the rotated MD snapshots, the corresponding orientation-dependent PDFs were calculated following the procedures described above. Then, the orientation-dependent PDFs calculated from the rotated MD snapshots were averaged for each unit angular vector. Using the averaged orientation-dependent PDFs $\left(g_{j k}^{a v e}\left(r, \overrightarrow{\boldsymbol{v}_{m}}\right)\right)$, the cage scattering pattern arising from the anisotropic orientation of the solute molecules was calculated with Eq. (13). Finally, isotropic and anisotropic cage terms were extracted from $\mathrm{S}_{\text {cage }}(\overrightarrow{\boldsymbol{q}})$ following the previously reported procedures ${ }^{33}$ as shown in Supplementary Fig. 8. More details are described in the section "Anisotropic cage term" of Supplementary Information.

Density functional theory calculation. Geometry optimization was performed using density functional theory (DFT) for $\mathrm{I}_{2}{ }^{-}$and $\mathrm{I}_{3}{ }^{-}$to obtain the molecular structure for initial cage calculations. We used the recently-developed $\omega \mathrm{B} 97 \mathrm{XD}$ functional ${ }^{53}$ as a DFT exchange-correlation functional. To treat the scalar relativistic effect of iodine, we used aug-cc-pVDZ-PP small-core relativistic effective core potential $(\mathrm{RECP})^{54}$. For other atoms $(\mathrm{C}, \mathrm{O}$, and $\mathrm{H}), 6-31++\mathrm{G}(\mathrm{d})$ basis sets were used. We also used the integral-equation-formalism polarizable continuum model (IEFPCM) method $^{55}$ to describe the solvent effect implicitly. We used the natural population analysis (NPA) for characterizing atomic charge. All DFT calculations were performed using the Gaussian16 program.

Molecular dynamics simulations. Molecular dynamics (MD) simulations were performed using the MOLDY program to obtain the cage terms for all the chemical species involved in the reaction ${ }^{56}$. One rigid solute molecule was embedded in a virtual cubic box of $\sim 32.5 \AA$ size containing 512 rigid methanol solvent molecules. The internal structure of each molecule was fixed, and the intermolecular interactions were governed by Coulomb forces and Lennard-Jones potentials. For describing the intermolecular interactions, we used a six-site all-atom (AA) model of methanol, OPLS-AA force field ${ }^{57,58}$. Structures of solute molecules $\left(\mathrm{I}_{3}{ }^{-}, \mathrm{I}_{2}{ }^{-}\right.$, and I•) were optimized using the DFT method, as written in the section "Density functional theory calculation" of Methods. All simulations were performed at an ambient temperature of $300 \mathrm{~K}$ with a solvent density of $0.792 \mathrm{~g} / \mathrm{cm}^{3}$. The system was equilibrated over $20 \mathrm{ps}$ via coupling to a Nose-Hoover thermostat ${ }^{59}$. The simulations were performed in the NVT ensemble with a time step of $0.1 \mathrm{fs}$, and the trajectories were followed up to $1 \mathrm{~ns}$.

Rotational dynamics simulation. The analysis of the experimental data reveals that the value of the rotational diffusion time constants of $\mathrm{I}_{3}{ }^{-}$and $\mathrm{I}_{2}{ }^{-}$are $13.5 \mathrm{ps}$ and 6.3 ps, respectively, as shown in Fig. 5. To support the experimental results, we conducted simulations to verify the rotational diffusion time constants of the intermediates 47,60 . The anisotropy at time $t, r(t)$, measures the correlation function of the second Legendre polynomial of the scalar product polarization direction and the orientation of molecules ${ }^{46}$ (transition dipole moments) at time delay, $t$.

$$
r(t)=A<P_{2}\left[\mu_{\text {pump }}(0) \mu_{\text {ori }}(t)\right]>=A<P_{2}[\cos \theta]>
$$

Here, $\theta$ is the angle between the pump polarization and the orientation of the molecule and $\mathrm{A}$ is the anisotropy of the molecule. Based on the equation above, we calculate the correlation function of $\mathrm{I}_{3}{ }^{-}$and the $\mathrm{I}_{2}{ }^{-}$by using the MD simulation assuming the orientation of the molecule is aligned to the polarization axis of the pump pulse. Details of the MD simulation is described in the section "Molecular dynamics simulations", noting that the parameters of the MD simulation used for the cage calculation were used identically. From the simulation, snapshots were extracted every $10 \mathrm{fs}$, and the unit vector of transition dipole moment (same with the unit vector of dissociating bond for $\mathrm{I}_{3}{ }^{-}$) was calculated. Because the rotational diffusion dynamics of the molecule should occur regardless of the initial condition, we calculated the correlation function of the molecules for every single step of the 
simulation and averaged the simulated data. Calculated correlation functions according to Eq. (14) are presented in Supplementary Fig. 11. The rotational diffusion time constant of $\mathrm{I}_{3}{ }^{-}$and $\mathrm{I}_{2}{ }^{-}$are calculated to be $15.8 \mathrm{ps}$ and $3.8 \mathrm{ps}$, respectively. The rotational diffusion time constants obtained from the simulation are consistent with those obtained from the analysis of the experimental data shown in Fig. 5 and those reported in previous studies ${ }^{45,46}$.

\section{Data availability}

The time-resolved isotropic and anisotropic difference scattering data analyzed in this study corresponding to Figs. 2c, 4a and 5a are provided as the Source Data file. All relevant data that support the findings of this study are available from the corresponding author upon request. Source data are provided with this paper.

\section{Code availability}

The codes used for the analysis here are available from the corresponding author on request.

Received: 11 August 2021; Accepted: 11 January 2022;

Published online: 26 January 2022

\section{References}

1. Bressler, C. et al. Femtosecond XANES study of the light-induced spin crossover dynamics in an iron(II) complex. Science 323, 489-492 (2009).

2. Vorobyev, V., Budkina, D. S. \& Tarnovsky, A. N. Femtosecond excited-state dynamics and nitric oxide photorelease in a prototypical ruthenium nitrosyl complex. J. Phys. Chem. 11, 4639-4643 (2020).

3. Miller, N. A. et al. Polarized XANES monitors femtosecond structural evolution of photoexcited vitamin B12. J. Am. Chem. Soc. 139, 1894-1899 (2017).

4. Cordones, A. A. et al. Transient metal-centered states mediate isomerization of a photochromic ruthenium-sulfoxide complex. Nat. Commun. 9, 1989 (2018).

5. Yoon, S.-J. et al. Multistimuli two-color luminescence switching via different slip-stacking of highly fluorescent molecular sheets. J. Am. Chem. Soc. 132, 13675-13683 (2010).

6. Kao, Y.-T., Saxena, C., Wang, L., Sancar, A. \& Zhong, D. Direct observation of thymine dimer repair in DNA by photolyase. Proc. Natl. Acad. Sci. USA 102, 16128 (2005).

7. Zhu, X., Farrer, R. A. \& Fourkas, J. T. Optical kerr effect spectroscopy using time-delayed pairs of pump pulses with orthogonal polarizations. J. Phys. Chem. B 109, 8481-8488 (2005).

8. Liu, Y. et al. A heteroleptic ferrous complex with mesoionic bis(1,2,3-triazol-5ylidene) ligands: taming the MLCT excited state of iron(II). Chem. Eur. J. 21, 3628-3639 (2015).

9. Kubin, M. et al. Probing the oxidation state of transition metal complexes: a case study on how charge and spin densities determine Mn L-edge X-ray absorption energies. Chem. Sci. 9, 6813-6829 (2018).

10. Haldrup, K. et al. Ultrafast X-ray scattering measurements of coherent structural dynamics on the ground-state potential energy surface of a diplatinum molecule. Phys. Rev. Lett. 122, 063001 (2019).

11. Kim, J. G. et al. Mapping the emergence of molecular vibrations mediating bond formation. Nature 582, 520-524 (2020).

12. van Driel, T. B. et al. Atomistic characterization of the active-site solvation dynamics of a model photocatalyst. Nat. Commun. 7, 13678 (2016).

13. Yang, J. et al. Imaging CF(3)I conical intersection and photodissociation dynamics with ultrafast electron diffraction. Science 361, 64-67 (2018).

14. Rimmerman, D. et al. Insulin hexamer dissociation dynamics revealed by photoinduced T-jumps and time-resolved X-ray solution scattering. Photochem. Photobiol. Sci. 17, 874-882 (2018).

15. Stankus, B. et al. Ultrafast X-ray scattering reveals vibrational coherence following Rydberg excitation. Nat. Chem. 11, 716-721 (2019).

16. Glownia, J. M. et al. Self-referenced coherent diffraction X-ray movie of ångstrom- and femtosecond-scale atomic motion. Phys. Rev. Lett. 117, 153003 (2016).

17. Ishikawa, T. et al. Direct observation of collective modes coupled to molecular orbital-driven charge transfer. Science 350, 1501-1505 (2015).

18. Arnlund, D. et al. Visualizing a protein quake with time-resolved X-ray scattering at a free-electron laser. Nat. Methods 11, 923-926 (2014).

19. Ihee, H. et al. Ultrafast $x$-ray diffraction of transient molecular structures in solution. Science 309, 1223-1227 (2005).

20. Choi, M. et al. Effect of the abolition of intersubunit salt bridges on allosteric protein structural dynamics. Chem. Sci. 12, 8207-8217 (2021).

21. Kim, T. W. et al. Protein folding from heterogeneous unfolded state revealed by time-resolved X-ray solution scattering. Proc. Natl. Acad. Sci. USA 117, 14996-15005 (2020).
22. Lee, S. J. et al. Reversible molecular motional switch based on circular photoactive protein oligomers exhibits unexpected photo-induced contraction. Cell Rep. Phys. Sci. 2, 100512 (2021).

23. Choi, E. H. et al. Filming ultrafast roaming-mediated isomerization of bismuth triiodide in solution. Nat. Commun. 12, 4732 (2021).

24. Lee, Y. et al. Ultrafast coherent motion and helix rearrangement of homodimeric hemoglobin visualized with femtosecond X-ray solution scattering. Nat. Commun. 12, 3677 (2021).

25. Kim, K. H. et al. Direct observation of bond formation in solution with femtosecond X-ray scattering. Nature 518, 385-389 (2015).

26. Coropceanu, V., Chen, X.-K., Wang, T., Zheng, Z. \& Brédas, J.-L. Charge-transfer electronic states in organic solar cells. Nat. Rev. Mater. 4, 689-707 (2019).

27. Cammarata, M. et al. Charge transfer driven by ultrafast spin transition in a CoFe Prussian blue analogue. Nat. Chem. 13, 10-14 (2021).

28. Josefsson, I. et al. Collective hydrogen-bond dynamics dictates the electronic structure of aqueous I3(-). Phys. Chem. Chem. Phys. 15, 20189-20196 (2013).

29. Zhang, F. S. \& Lynden-Bell, R. M. Solvent-induced symmetry breaking. Phys. Rev. Lett. 90, 185505 (2003).

30. Jena, N. K. et al. Solvent-dependent structure of the I3- ion derived from photoelectron spectroscopy and ab initio molecular dynamics simulations. Chem. Eur. J. 21, 4049-4055 (2015).

31. Kim, K. H. et al. Global reaction pathways in the photodissociation of I3 (-) ions in solution at 267 and $400 \mathrm{~nm}$ studied by picosecond X-ray liquidography. Chemphyschem 14, 3687-3697 (2013).

32. Baskin, J. S. \& Zewail, A. H. Oriented ensembles in ultrafast electron diffraction. Chemphyschem 7, 1562-1574 (2006).

33. Biasin, E. et al. Anisotropy enhanced X-ray scattering from solvated transition metal complexes. J. Synchrotron Radiat. 25, 306-315 (2018).

34. Kim, T. K., Lee, J. H., Wulff, M., Kong, Q. \& Ihee, H. Spatiotemporal kinetics in solution studied by time-resolved X-ray liquidography (solution scattering). Chemphyschem 10, 1958-1980 (2009).

35. Kim, K. H. et al. Solvent-dependent molecular structure of ionic species directly measured by ultrafast x-ray solution scattering. Phys. Rev. Lett. 110, 165505 (2013).

36. Biasin, E. et al. Direct observation of coherent femtosecond solvent reorganization coupled to intramolecular electron transfer. Nat. Chem. 13, 343-349 (2021)

37. Berntsson, O. et al. Time-resolved X-ray solution scattering reveals the structural photoactivation of a light-oxygen-voltage photoreceptor. Structure 25, 933-938.e933 (2017).

38. Ravishankar, H. et al. Tracking $\mathrm{Ca}(2+)$ ATPase intermediates in real time by $\mathrm{x}$-ray solution scattering. Sci. Adv. 6, eaaz0981 (2020).

39. Sato, H., Hirata, F. \& Myers, A. B. Theoretical study of the solvent effect on triiodide ion in solutions. J. Phys. Chem. A 102, 2065-2071 (1998).

40. Johnson, A. E. \& Myers, A. B. Solvent effects in the raman spectra of the triiodide ion: observation of dynamic symmetry breaking and solvent degrees of freedom. J. Phys. Chem. 100, 7778-7788 (1996).

41. Vala, J., Kosloff, R. \& Harvey, J. N. Ab initio and diatomics in molecule potentials for I2-, I2, I3-, and I3. J. Chem. Phys. 114, 7413-7423 (2001).

42. Ledbetter, K. et al. Photodissociation of aqueous I3- observed with liquidphase ultrafast mega-electron-volt electron diffraction. Struct. Dyn. 7, 064901 (2020).

43. Hess, S., Bürsing, H. \& Vöhringer, P. Dynamics of fragment recoil in the femtosecond photodissociation of triiodide ions in liquid solution. J. Chem. Phys. 111, 5461-5473 (1999).

44. Panman, M. R. et al. Observing the structural evolution in the photodissociation of diiodomethane with femtosecond solution X-ray scattering. Phys. Rev. Lett. 125, 226001 (2020).

45. Kühne, T. \& Vöhringer, P. Transient anisotropy and fragment rotational excitation in the femtosecond photodissociation of triiodide in solution. $J$ Phys. Chem. A 102, 4177-4185 (1998).

46. Banin, U. \& Ruhman, S. Ultrafast photodissociation of I3. Coherent photochemistry in solution. J. Chem. Phys. 98, 4391-4403 (1993).

47. Banerjee, P., Yashonath, S. \& Bagchi, B. Rotation driven translational diffusion of polyatomic ions in water: A novel mechanism for breakdown of StokesEinstein relation. J. Chem. Phys. 146, 164502 (2017).

48. James, F. \& Roos, M. Minuit - a system for function minimization and analysis of the parameter errors and correlations. Comput. Phys. Commun. 10 343-367 (1975).

49. Kjær, K. S. et al. Introducing a standard method for experimental determination of the solvent response in laser pump, X-ray probe timeresolved wide-angle X-ray scattering experiments on systems in solution. Phys. Chem. Chem. Phys. 15, 15003-15016 (2013).

50. Cammarata, M. et al. Impulsive solvent heating probed by picosecond $\mathrm{x}$-ray diffraction. J. Chem. Phys. 124, 124504 (2006).

51. Ki, H., Lee, Y., Choi, E. H., Lee, S. \& Ihee, H. SVD-aided non-orthogonal decomposition (SANOD) method to exploit prior knowledge of spectral components in the analysis of time-resolved data. Struct. Dyn. 6, 024303 (2019). 
52. Koay, C. G. Analytically exact spiral scheme for generating uniformly distributed points on the unit sphere. J. Comput. Sci. 2, 88-91 (2011).

53. Chai, J. D. \& Head-Gordon, M. Long-range corrected hybrid density functionals with damped atom-atom dispersion corrections. Phys. Chem. Chem. Phys. 10, 6615-6620 (2008).

54. Peterson, K. A., Shepler, B. C., Figgen, D. \& Stoll, H. On the spectroscopic and thermochemical properties of $\mathrm{ClO}, \mathrm{BrO}, \mathrm{IO}$, and their anions. J. Phys. Chem. A 110, 13877-13883 (2006).

55. Cancès, E., Mennucci, B. \& Tomasi, J. A new integral equation formalism for the polarizable continuum model: Theoretical background and applications to isotropic and anisotropic dielectrics. J. Chem. Phys. 107, 3032-3041 (1997).

56. Refson, K. Moldy: a portable molecular dynamics simulation program for serial and parallel computers. Comput. Phys. Commun. 126, 310-329 (2000).

57. Jorgensen, W. L., Ulmschneider, J. P. \& Tirado-Rives, J. Free energies of hydration from a generalized born model and an all-atom force field. J. Phys. Chem. B 108, 16264-16270 (2004).

58. Zangi, R. Refinement of the OPLSAA force-field for liquid alcohols. ACS Omega 3, 18089-18099 (2018).

59. Hoover, W. G. Canonical dynamics: equilibrium phase-space distributions. Phys. Rev. A 31, 1695-1697 (1985).

60. Volk, M. et al. Anisotropy measurements of solvated HgI2 dissociation: transition state and fragment rotational dynamics. J. Phys. Chem. A 101, 638-643 (1997).

\section{Acknowledgements}

This work was supported by the Institute for Basic Science (IBS-R033). The experiments were performed at the XSS of PAL-XFEL (proposal nos. 2019-2nd-XSS-013 and 2020-2ndXSS-001). The authors thank J. H. Lee, J. Park, I. Eom, S. H. Chun, S. Kim, S. Nozawa, T. Sato, R. Fukaya, S. Adachi, T. W. Kim, S. J. Lee, Y., Lee, H. Kim, C. W. Ahn, Y. Cha, S. Eom, J. Gu, D. Kim, K. W. Lee, J. Kang, S. Choi, and J. Kim for their help in optimizing experimental conditions, and J. Kim and H. Kim for their help in DFT calculations.

\section{Author contributions}

H.I. supervised the project; H.I. conceived the idea and designed the experiment; J.H., J.G.K., D.-S.A., and H.I. developed data analysis strategy; J.H, J.G.K., E.H.C., H.K., J.K.,
S.L., and H.I. performed the experiment; J.H. analyzed the data; J.H., J.G.K., and H.I wrote the manuscript with contributions from all authors.

\section{Competing interests}

The authors declare no competing interests.

\section{Additional information}

Supplementary information The online version contains supplementary material available at https://doi.org/10.1038/s41467-022-28168-0.

Correspondence and requests for materials should be addressed to Hyotcherl Ihee.

Peer review information Nature Communications thanks the anonymous reviewers for their contribution to the peer review of this work.

Reprints and permission information is available at http://www.nature.com/reprints

Publisher's note Springer Nature remains neutral with regard to jurisdictional claims in published maps and institutional affiliations.

(c) (i) Open Access This article is licensed under a Creative Commons Attribution 4.0 International License, which permits use, sharing, adaptation, distribution and reproduction in any medium or format, as long as you give appropriate credit to the original author(s) and the source, provide a link to the Creative Commons license, and indicate if changes were made. The images or other third party material in this article are included in the article's Creative Commons license, unless indicated otherwise in a credit line to the material. If material is not included in the article's Creative Commons license and your intended use is not permitted by statutory regulation or exceeds the permitted use, you will need to obtain permission directly from the copyright holder. To view a copy of this license, visit http://creativecommons.org/ licenses/by/4.0/.

(C) The Author(s) 2022 